\title{
ARTICLE Multiple circulating saponins from intravenous ShenMai inhibit OATP1Bs in vitro: potential joint precipitants of drug interactions
}

\author{
Olajide E. Olaleye ${ }^{1,2}$, Wei Niu ${ }^{1}$, Fei-fei $\mathrm{Du}^{1}$, Feng-qing Wang ${ }^{1}$, Fang $\mathrm{Xu}^{1}$, Salisa Pintusophon ${ }^{1}$, Jun-lan $\mathrm{Lu}^{1}$, Jun-ling Yang ${ }^{1}$ and $\mathrm{Chuan} \mathrm{Li}^{1,2}$
}

ShenMai, an intravenous injection prepared from steamed Panax ginseng roots (Hongshen) and Ophiopogon japonicus roots (Maidong), is used as an add-on therapy for coronary artery disease and cancer; saponins are its bioactive constituents. Since many saponins inhibit human organic anion-transporting polypeptides (OATP)1B, this investigation determined the inhibition potencies of circulating ShenMai saponins on the transporters and the joint potential of these compounds for ShenMai-drug interaction. Circulating saponins and their pharmacokinetics were characterized in rats receiving a 30-min infusion of ShenMai at $10 \mathrm{~mL} / \mathrm{kg}$. Inhibition of human OATP1B1/1B3 and rat Oatp1b2 by the individual saponins was investigated in vitro; the compounds' joint inhibition was also assessed in vitro and the data was processed using the Chou-Talalay method. Plasma protein binding was assessed by equilibrium dialysis. Altogether, 49 saponins in ShenMai were characterized and graded into: 10-100 $\mu \mathrm{mol} / \mathrm{day}$ (compound doses from ShenMai; 7 compounds), 1-10 $\mu \mathrm{mol} / \mathrm{day}$ (17 compounds), and $<1 \mu \mathrm{mol} /$ day ( 25 compounds, including Maidong ophiopogonins). After dosing, circulating saponins were protopanaxadiol-type ginsenosides $R b_{1}, R b_{2}, R c, R d, R a_{1}, R g_{3}, R a_{2}$, and $\mathrm{Ra}_{3}$, protopanaxatriol-type ginsenosides $R g_{1}, R e, R g_{2}$, and $R f$, and ginsenoside Ro. The protopanaxadiol-type ginsenosides exhibited maximum plasma concentrations of $2.1-46.6 \mu \mathrm{mol} / \mathrm{L}$, plasma unbound fractions of $0.4-1.0 \%$ and terminal half-lives of $15.6-28.5 \mathrm{~h}$ (ginsenoside $\mathrm{Rg}_{3}, 1.9 \mathrm{~h}$ ), while the other ginsenosides exhibited $0.1-7.7 \mu \mathrm{mol} / \mathrm{L}, 20.8-99.2 \%$, and $0.2-0.5 \mathrm{~h}$, respectively. The protopanaxadiol-type ginsenosides, ginsenosides without any sugar attachment at C-20 (except ginsenoside Rf), and ginsenoside Ro inhibited OATP1B3 more potently $\left(\mathrm{IC}_{50}, 0.2-3.5 \mu \mathrm{mol} / \mathrm{L}\right)$ than the other ginsenosides $(\geq 22.6 \mu \mathrm{mol} / \mathrm{L})$. Inhibition of OATP1B1 by ginsenosides was less potent than OATP1B3 inhibition. Ginsenosides $R b_{1}, R b_{2}, R c, R d, R o, R a_{1}, R e$, and $R g_{2}$ likely contribute the major part of OATP1B3-mediated ShenMai-drug interaction potential, in an additive and time-related manner.

Keywords: ShenMai injection; organic anion-transporting polypeptide 1B; drug interactions; ginsenoside; herbal medicine

Acta Pharmacologica Sinica (2019) 40:833-849; https://doi.org/10.1038/s41401-018-0173-9

\section{INTRODUCTION}

In China, many herbal medicines have been incorporated into synthetic drug-based treatment of multifactorial diseases, such as cardiovascular diseases, cancer, and infectious diseases, for therapeutic advantage, either because their therapeutic effects are complementary and/or synergistic or because such effects can be achieved with reduced drug side effects or with reduced drug resistance [1-7]. However, substances from co-administered herbal products and synthetic drugs have potential for untoward pharmacokinetic interactions [8-11]. Constituents of many botanical products, evaluated as precipitants of drug interactions, have been shown to inhibit and/or induce drug metabolizing enzymes and transporters in vitro [12-16]. In addition, an herbal medicine may also be the object of drug interactions [17]. In China, herbal medicines are extensively used in clinics as multi-compound extracts and highly purified compounds, and are prescribed by both physicians practicing Western medicine and those practicing Chinese traditional medicine. Like the oral herbal tablets and capsules, herbal injections for intravenous administration are also extensively used in clinics. By delivering its bioactive constituents directly into the bloodstream and bypassing the enterohepatic barrier, such injection often facilitates high levels of exposure to these compounds for rapid effect. Given the common comedication of Chinese herbal medicines with synthetic medicines, drug interaction information is essential. This will help ensure pharmacokinetic compatibility (i.e., absence of any undesirable, unintentional, or unmanageable pharmacokinetic interaction that can lead to loss of drug effectiveness and safety) for the success of such combination therapies.

Chinese herbal medicines often contain multiple bioactive constituents bearing similar structures. These herbal compounds could interact with the same drug metabolizing enzymes and/or transporters (interacting proteins), and might jointly cause drug interactions, to which they contribute to varying extents. Here, an approach is proposed for investigating the drug interaction potential of an herbal medicine: (1) characterizing the medicine's

\footnotetext{
${ }^{1}$ State Key Laboratory of Drug Research, Shanghai Institute of Materia Medica, Chinese Academy of Sciences, Shanghai 201203, China and ${ }^{2}$ University of Chinese Academy of Sciences, Shanghai 201203, China

Correspondence: Chuan Li (chli@simm.ac.cn) or Jun-ling Yang (yangjl@simm.ac.cn)

Jun-lan Lu was a visiting student from Tianjin University of Traditional Chinese Medicine, Tianjin 301617, China.
}

Received: 4 July 2018 Accepted: 14 September 2018

Published online: 16 October 2018 
chemical composition; (2) identifying its circulating compounds that are bioavailable for such an interaction; (3) assessing the modulation potencies of these herbal compounds on the relevant interacting proteins; (4) characterizing their pharmacokinetic characteristics that can influence the compounds' contributions to the interaction potential; and (5) evaluating the joint modulation of the same interacting proteins by the identified compounds. Data obtained from such investigation will inform model-based prediction and clinical studies of drug interaction related to the medicine.

ShenMai injection, an intravenous botanical drug product prepared from steamed Panax ginseng roots (Hongshen) and Ophiopogon japonicus roots (Maidong), is approved by the China Food and Drug Administration (China FDA) as add-on therapy in treatment of coronary artery disease and cancer. In a recent double-blind, multicenter, placebo-controlled, prospective, randomized clinical trial in 240 patients with chronic heart failure and coronary artery disease, adding ShenMai $(100 \mathrm{~mL} /$ day, for 7 days) to standard treatment yielded greater improvements in New York Heart Association functional classification (the primary endpoint) and also in 6-min walking distance, short-form 36 health survey score, and TCM syndrome scores than the standard treatment alone; adding ShenMai was well-tolerated, with no apparent safety concerns [18]. Also, clinical studies have provided evidence that adding ShenMai alleviates chemotherapy-induced side effects in patients with breast cancer or with non-small cell lung cancer $[1,19]$. Saponins, the bioactive constituents of ShenMai, are believed to be responsible for the injection's therapeutic action [20-23]. Saponins from Hongshen are triterpene saponins, which are classified here into 20(S)-protopanaxadiol-type (ppd-type) ginsenosides, 20(S)-protopanaxatriol-type (ppt-type) ginsenosides, and ginsenosides of other types, while those from Maidong are steroidal saponins. Many triterpene and steroidal saponins have been found to be inhibitors or substrates of the human hepatic uptake transporters organic anion-transporting polypeptide (OATP) 1B1 and/or OATP1B3 $[14,17,24,25]$. Given that ShenMai is commonly coadministered with other medicines, understanding its potential for drug interactions is essential for its rational clinical use. This investigation was designed to evaluate OATP1B-mediated ShenMai-drug interaction potential by identifying ShenMai saponins that are likely to contribute the major part of such interactions and their joint inhibition of the transporters.

\section{MATERIALS AND METHODS}

Materials and chemicals

ShenMai injection (lot number, 161210D2, 17010432, 170515D2) was manufactured by Shineway Pharmaceutical Group (Shijiazhuang, China) with a China FDA drug ratification number of GuoYaoZhunZi-Z13020888. ShenMai from the lot 161210D2 was used in the current rat studies (Supplementary Table S1). Each milliliter of the injection is prepared from $0.1 \mathrm{~g}$ each of Hongshen (steamed $P$. ginseng roots) and Maidong ( $O$. japonicus roots), yielding an herb-to-injection ratio of 1:5. The final product is a sterile and nonpyrogenic injection for intravenous administration. Each milliliter of ShenMai is standardized to contain not $<0.10 \mathrm{mg}$ total of ginsenoside $\mathrm{Rg}_{1}$ and ginsenoside $\mathrm{Re}$; not $<0.10 \mathrm{mg}$ ginsenoside $\mathrm{Rb}_{1}$; and $0.20-0.90 \mathrm{mg}$ total of ginsenoside $\mathrm{Rg}_{1}$, ginsenoside $\mathrm{Re}$ and ginsenoside $\mathrm{Rb}_{1}$. Crude samples of the component herbs Hongshen (steamed $P$. ginseng roots) and Maidong (O. japonicus roots) were also obtained from Shineway Pharmaceutical Group and were stored at $-20^{\circ} \mathrm{C}$ until analysis.

Ginsenosides $\mathrm{Rb}_{1}, \mathrm{Rb}_{2}, \mathrm{Rg}_{3}, \mathrm{~F}_{1}, \mathrm{~F}_{2}, \mathrm{Rg}_{1}, \mathrm{Re}, \mathrm{Rg}_{2}, \mathrm{Rf}$, and $\mathrm{Rh}_{1}$, compound-K, 20(S)-protopanaxadiol, and 20(S)-protopanaxatriol were obtained from the National Institutes for Food and Drug Control (Beijing, China); ginsenosides $\mathrm{Rg}_{6}, \mathrm{Rk}_{1}$, and $\mathrm{Rg}_{5}$, and ophiopogonin $\mathrm{D}^{\prime}$, from Push Bio-technology (Chengdu, China); ginsenosides Ro and $\mathrm{Rh}_{4}$, and notoginsenoside $\mathrm{R}_{1}$, from Chroma Biotechnology (Chengdu, China); ginsenosides $\mathrm{Ra}_{1}$ and $\mathrm{Ra}_{2}$, from Star Ocean Ginseng Bio-pharmaceutical (Shanghai, China); ginsenosides Rc and Rd, and ophiopogonin D, from Tauto Biotech (Shanghai, China); and 20-gluco-ginsenoside Rf, from Fengshanjian (Kunming, China). The purity of these compounds exceeded 98\%. Rifampin, HPLC grade methanol, formic acid, and lithium acetate dihydrate were obtained from Sigma-Aldrich (St Louis, MO, USA). Estradiol-17 $\beta$-D-glucuronide $\left(E_{2} 17 \beta G\right)$ was obtained from Cayman Chemical (Ann Arbor, MI, USA). Deionized water was purified using a Millipore Milli-Q Integral 3 cabinet water purifying system (Milford, MA, USA).

Pentobarbital was obtained from Shanghai Westang Biotechnology (Shanghai, China) and sodium taurocholate, from Solarbio Life Sciences (Beijing, China). Human liver microsomes and human liver cytosol were obtained from Corning Gentest (Woburn, MA, USA). Rat liver homogenate and rat liver microsomes were prepared in-house [26]. Human embryonic kidney 293 (HEK-293) cells were obtained from American Type Culture Collection (Manassas, VA, USA). Human OATP1B1 and OATP1B3 and rat Oatp 1 b2 expression plasmids were constructed by cloning human OATP1B1 (NM_006446) and OATP1B3 (NM_019844) and rat Oatp1b2 (NM_031650) open reading frames into expression vector pcDNA 3.1(+) by Invitrogen Life Technologies (Shanghai, China). Before use, the expression plasmids were sequenceverified and functionally characterized [14]. The cell culture reagents were obtained from Invitrogen Life Technologies.

\section{In vivo rat studies of ShenMai}

All animal care and use complied with the Guidance for Ethical Treatment of Laboratory Animals (The Ministry of Science and Technology of China, 2006, at www.most.gov.cn/fggw/zfwj/ zfwj2006). Rat studies were implemented according to protocols that were reviewed and approved by the Institutional Animal Care and Use Committee at Shanghai Institute of Materia Medica (Shanghai, China). Rat studies are reported in accordance with the ARRIVE guidelines [27, 28]. Male Sprague-Dawley rats were obtained from SIPPR-BK Laboratory Animal Co. Ltd. (Shanghai, China), housed at $20-24{ }^{\circ} \mathrm{C}$ and relative humidity of $30-70 \%$ with a 12-h light/dark cycle, and maintained under specific-pathogenfree conditions. Rats were provided commercial rat chow and access to filtered tap water ad libitum and were acclimated to the facilities and environment for one week before use. All rats received in-house femoral vein cannulation for infusion of ShenMai, while some received, in addition, femoral-arterycannulation for blood sampling or bile duct cannulation for bile sampling. After surgery, rats were housed singly and allowed to regain their preoperative body weights before the studies. All used rats were killed with $\mathrm{CO}_{2}$ gas. A total of 18 rats were used in this investigation for three studies.

To assess the systemic exposure to saponins, six rats received a 30 -min intravenous infusion of ShenMai at $10 \mathrm{~mL} / \mathrm{kg}$; the dose was translated from the label human dose of ShenMai $(100 \mathrm{~mL} /$ day) by using the body surface area normalization method [29]. Serial blood samples [around $150 \mu \mathrm{L}$; before and 15, 30 (just before terminating the infusion), 35, $45 \mathrm{~min}$, and 1, 1.5, 2.5, 4.5, 8.5, 24, 48, and $72 \mathrm{~h}$ after starting the infusion] were collected in heparinized tubes and then centrifuged to yield plasma fractions. The plasma samples were aliquoted and then stored at $-70^{\circ} \mathrm{C}$ pending analysis.

To investigate renal excretion of saponins, six rats that were housed singly in metabolic cages with urine collection tubes frozen at $-15^{\circ} \mathrm{C}$ received a $30-\mathrm{min}$ intravenous infusion of ShenMai at $10 \mathrm{~mL} / \mathrm{kg}$ and urine samples were collected before and $0-8,8-24,24-48$, and $48-72 \mathrm{~h}$ after starting the infusion. The urine samples collected were weighed, aliquoted, and then stored at $-70^{\circ} \mathrm{C}$ pending analysis.

To investigate hepatobiliary excretion of saponins, six rats received a 30-min intravenous infusion of ShenMai at $10 \mathrm{~mL} / \mathrm{kg}$. 
Serial bile samples were collected before and $0-8,8-24,24-32$, $32-48,48-56$, and $56-72 \mathrm{~h}$ after starting the infusion of ShenMai. During bile collection, a solution of sodium taurocholate $(7.68 \mathrm{mg} / \mathrm{mL}$ in saline; $\mathrm{pH} 7.4)$ was infused $(1 \mathrm{~mL} / \mathrm{h})$ via the duodenum. The bile samples collected were weighed, aliquoted, and then stored at $-70^{\circ} \mathrm{C}$ pending analysis.

Determination of unbound fractions of ShenMai ginsenosides in plasma

To determine the compounds' unbound fractions in plasma, freshly collected blank rat and human plasma samples were spiked separately with test ginsenosides at $37^{\circ} \mathrm{C}$ and equilibrium dialysis using Spectra/Por 2 dialysis membranes (molecular weight cutoff, 12-14 kDa; Rancho Dominguez, CA, USA) was performed [30]. After equilibrating for $24 \mathrm{~h}$ at $55 \mathrm{rpm}$ and $37^{\circ} \mathrm{C}$, the dialysate and the plasma were sampled for analysis. The test ginsenosides were ginsenosides $R b_{1}, R b_{2}, R c, R d, R a_{1}, R g_{3}, R a_{2}, R a_{3}, R g_{1}, R e, R g_{2}$, $R f, R h_{1}, R o, R g_{6}, R k_{1}, R h_{4}$, and $R_{5}$, notoginsenoside $R_{1}$, and 20gluco-ginsenoside $\mathrm{Rf}$, all of which were tested at $5 \mu \mathrm{mol} / \mathrm{L}$, except for the ppd-type ginsenosides and ginsenosides $\mathrm{Rk}_{1}$ and $\mathrm{Rg}_{5}$ also at $50 \mu \mathrm{mol} / \mathrm{L}$. Collection of blank blood from healthy human volunteers was approved by the Ethics Committee for Biomedical Research at Shanghai Institute of Materia Medica. Blood samples were heparinized and then centrifuged to yield plasma fractions, which were put together to yield pooled blank human plasma and pooled blank rat plasma. Unbound fraction in plasma $\left(f_{\mathrm{u}-\text { plasma }}\right)$ was calculated using the following equation:

$f_{\mathrm{u}-\text { plasma }}=\left(C_{\mathrm{d}} / C_{\mathrm{p}}\right) \times 100 \%$,

where $C_{d}$ is the concentration in the dialysate sample and $C_{p}$ is the corresponding concentration in the post-dialysis plasma sample.

In vitro metabolism studies of ShenMai saponins

To better understand whether systemic exposure to ginsenoside $\mathrm{Rd}$ (of ppd-type) could be influenced by (besides its own dose from ShenMai) other co-existing ppd-type ginsenosides' metabolism in rats and whether a similar scenario could take place in humans, ginsenosides $R b_{1}, R b_{2}, R c, R a_{1}, R a_{2}$, and $R a_{3}$ were separately incubated in triplicate, at a final substrate concentration of $10 \mu \mathrm{mol} / \mathrm{L}$, with rat liver homogenate at $37^{\circ} \mathrm{C}$ for $20 \mathrm{~h}$. The incubation samples were analyzed by liquid chromatography/ mass spectrometry to determine formation of ginsenoside Rd. In addition, such incubation was also performed using human liver cytosol for these ppd-type ginsenosides at a final substrate concentration of $10 \mu \mathrm{mol} / \mathrm{L}$.

Ginsenosides $\mathrm{Rg}_{3}, \mathrm{Rg}_{2}, \mathrm{Rf}$, and $\mathrm{Rh}$, share a structural feature, i.e., possessing no sugar attachment at $\mathrm{C}-20$. To better understand whether systemic exposure of these ginsenosides could be influenced by (in addition to their own doses from ShenMai) their metabolism in rats and whether a similar scenario could take place in humans, these four ginsenosides were separately incubated with rat liver microsomes in the presence of the cofactor reduced $\beta$-nicotinamide adenine dinucleotide phosphate (NADPH) using the method by $\mathrm{Hu}$ et al. [26]. In brief, the incubation was performed in triplicate at a final substrate concentration of $10 \mu \mathrm{mol} / \mathrm{L}$ and $37^{\circ} \mathrm{C}$ for $2 \mathrm{~h}$; the incubation samples were analyzed by liquid chromatography/mass spectrometry to determine formation of the oxidized metabolites. In addition, such incubation was also performed using human liver microsomes for these ginsenosides at a final substrate concentration of $10 \mu \mathrm{mol} / \mathrm{L}$.

In vitro assessment of interactions of ShenMai saponins with OATP1B1 and OATP1B3

Cell culture was conducted and uptake of ginsenosides $R b_{1}, R b_{2}$, $\mathrm{Rc}, \mathrm{Rd}, \mathrm{Ra}_{1}, \mathrm{Rg}_{3}, \mathrm{Ra}_{2}, \mathrm{Ra}_{3}, \mathrm{Rg}_{1}, \mathrm{Re}, \mathrm{Rg}_{2}, \mathrm{Rf}, \mathrm{Rh}_{1}, \mathrm{Ro}, \mathrm{Rg}_{6}, \mathrm{Rk}_{1}, \mathrm{Rh}_{4}$, and $R_{5}$, and 20-gluco-ginsenoside Rf, notoginsenoside $R_{1}$, and ophiopogonins $D$ and $D^{\prime}$ in HEK-293 cells transiently transfected with OATP1B1 or OATP1B3 were investigated using methods described by Jiang et al. [14]. The transport rate of the test compound in $\mathrm{pmol} / \mathrm{min} / \mathrm{mg}$ protein was calculated using the following equation:

Transport $=\left(C_{\mathrm{L}} \times V_{\mathrm{L}}\right) / T / W_{\mathrm{L}}$,

where $C_{\mathrm{L}}$ is the concentration in the cellular lysate, $V_{\mathrm{L}}$ is the volume of the lysate, $T$ is the incubation time $(10 \mathrm{~min})$, and $W_{\mathrm{L}}$ is the measured protein amount in the lysate $(\mathrm{mg})$. Differential uptake between the transfected cells (TC) and mock cells (MC) was defined as a net transport ratio (Transport ${ }_{\mathrm{TC}} /$ Transport $_{\mathrm{MC}}$ ratio). A net transport ratio $>3$ was taken to indicate that the test saponin was a substrate of the transporter. Before use, the transfected cells were functionally characterized by measuring uptake of $E_{2} 17 \beta G$; the net transport ratios were $55.3 \pm 3.8$ and $42.1 \pm 4.4$ for OATP1B1 and OATP1B3, respectively, in the absence of rifampin and $1.4 \pm 0.0$ and $1.0 \pm 0.0$, respectively, in the presence of rifampin $(100 \mu \mathrm{mol} / \mathrm{L})$. Inhibition potencies of the test saponins on the cellular transport of $E_{2} 17 \beta G$ by human OATP1B1 or OATP1B3 were initially screened at a saponin concentration of $100 \mu \mathrm{mol} / \mathrm{L}$. Half-maximal inhibitory concentrations $\left(I C_{50}\right)$ were then determined for those saponins demonstrating $>50 \%$ inhibition. The concentrations of $E_{2} 17 \beta G$ were 10 and $15 \mu \mathrm{mol} / \mathrm{L}$ for OATP1B1 and OATP1B3, respectively, while the concentrations of each test ginsenoside are shown in Supplementary Table S2. To assess the influence of 1-h preincubation with test ginsenosides (in the absence of the substrate $E_{2} 17 \beta G$ ) on their OATP1B1/1B3 inhibition, $I C_{50}$ values measured with such preincubation were compared with the respective $I C_{50}$ values measured without preincubation. Unbound fractions in incubation $\left(f_{\text {u-incubation }}\right)$ containing mock cells were measured for test ginsenosides at $10 \mu \mathrm{mol} / \mathrm{L}$, using a method by Posada et al. [31]. The mean $f_{\mathrm{u} \text {-incubation }}$ values of test ginsenosides were 96-114\%, except for ginsenosides $\mathrm{Rg}_{6}, \mathrm{Rk}_{1}$, and $\mathrm{Rg}_{5}$ with such values of $39-76 \%$.

In vitro assessment of joint inhibition of OATP1B1/1B3 by ginsenosides

Ginsenosides $R b_{1}, R b_{2}, R c, R d, R e$, and Ro were selected for investigation of their joint inhibition of OATP1B1 and OATP1B3. These selected ginsenosides exhibited the highest $\left(C_{30 \mathrm{~min}} \times\right.$ $f_{\mathrm{u} \text {-plasma }}$ ) $/ \mathrm{I} C_{50}$ ratios (where $C_{30 \mathrm{~min}}$ is the maximum rat plasma concentration at $30 \mathrm{~min}$ after starting 30 - $\mathrm{min}$ infusion, $f_{\mathrm{u} \text {-plasma }}$ is for human plasma, and $\mathrm{IC}_{50}$ is for the human OATP1B1/1B3). Joint inhibition was assessed using the Chou-Talalay method [32, 33]. Two combinations were tested, i.e., six ginsenosides $R b_{1}, R b_{2}, R c$, $\mathrm{Rd}, \mathrm{Re}$, and Ro mixed in a ratio similar to that of their " $C_{30 \mathrm{~min}} \times f_{\mathrm{u}}$ plasma" and four ppd-type ginsenosides $\mathrm{Rb}_{1}, \mathrm{Rb}_{2}, \mathrm{Rc}$, and $\mathrm{Rd}$ mixed in a ratio similar to that of their " $C_{1.5 \mathrm{~h}} \times f_{\mathrm{u} \text {-plasma" }}$ (where $C_{1.5 \mathrm{~h}}$ is rat plasma concentration at $1.5 \mathrm{~h}$ after starting 30 -min infusion). These ratios were held constant, while the total concentrations were varied by serial dilution. Joint inhibition of OATP1B1 and that of OATP1B3 by the ginsenoside combinations were assessed using the transiently transfected HEK-293 cells, with $E_{2} 17 \beta G$ as substrate. The joint inhibition was characterized with respect to combination index $(\mathrm{Cl})$, which was calculated using Combosyn Compusyn software (version 1.0; Paramus, NJ, USA). A Cl value of $<0.9,0.9-1.1$, or $>1.1$ was taken to indicate that the joint inhibition is synergistic, additive, or antagonistic respectively, i.e., producing a joint inhibition greater than, equal to, or lower than, respectively, the sum of inhibition by the individual compounds.

Characterization and grading of saponins present in ShenMai A Waters Synapt G2 high definition time-of-flight mass spectrometer (Manchester, UK), interfaced via a LockSpray source with 
a Waters Acquity ultra performance liquid chromatographic separation module (Milford, MA, USA), was used for detection, characterization, and grading of saponins present in ShenMai. Samples of the herbal injection were prepared by diluting with water, while samples of the component herbs Hongshen and Maidong were prepared by extracting with 50\% methanol followed by filtration. Chromatographic separation was achieved on a $1.7 \mu \mathrm{m}$ Waters BEH C18 column $\left(100 \mathrm{~mm} \times 2.1 \mathrm{~mm}\right.$ i.d.; $45^{\circ} \mathrm{C}$; Dublin, Ireland) with a mobile phase, delivered at $0.3 \mathrm{~mL} / \mathrm{min}$, consisting of $(A)$ methanol/water $(1: 99, \mathrm{v} / \mathrm{v}$; containing $1 \mathrm{mmol} / \mathrm{L}$ formic acid) and (B) methanol/water (99:1, v/v; containing $1 \mathrm{mmol} /$ $L$ formic acid). A 42-min gradient program was used, consisting of $0-2 \mathrm{~min}$ at $2 \% \mathrm{~B}, 2-32 \mathrm{~min}$ from $2 \%$ to $98 \% \mathrm{~B}, 32-37 \mathrm{~min}$ at $98 \% \mathrm{~B}$, and $37-42 \mathrm{~min}$ at $2 \% \mathrm{~B}$. The mass spectrometer was operated in resolution mode that gave a resolution power of around 20,000 and was externally calibrated over a range of $\mathrm{m} / \mathrm{z}$ $50-1500$ using a $5 \mathrm{mmol} / \mathrm{L}$ sodium formate solution at $10 \mu \mathrm{L} / \mathrm{min}$ and mass shifts during acquisition were corrected using leucine encephalin ( $m / z 554.2615$ for the negative ion mode). $\mathrm{MS}^{\mathrm{E}}$ data acquisition (in centroid mode, $\mathrm{m} / \mathrm{z}$ 50-1500) was achieved simultaneously using a trap collision energy of $3 \mathrm{~V}$ and a trap collision energy ramp of $30-50 \mathrm{~V}$ with a scan time of $0.4 \mathrm{~s}$.

Prior to analysis, information on saponins originating from Hongshen (steamed $P$. ginseng roots) and Maidong ( $O$. japonicus roots) was obtained by literature mining, and four literature references by Xie et al. [34], Yang et al. [35], Shin et al. [36], and $\mathrm{Li}$ et al. [37] gave the most comprehensive information about chemical constituents of these herbs and about chemotransformation of ginsenosides related to pharmaceutical processing. Saponins present in ShenMai were detected in the negative ion mode using an analyte-targeted detection approach, based on a compound list with information (obtained via the preanalysis literature mining) such as their names, structures, accurate molecular masses, electrospray ionization patterns, and collision-induced dissociation patterns. Those detected compounds that were suspected to be ShenMai saponins were characterized by comparing their accurate molecular masses, fragmentation profiles, and chromatographic retention times with those of the associated reference standards. When such standards were not available, characterization was based on comparison with the reported mass data for the suspected saponins and their reported chromatographic elution order with other related compounds. Grading of the characterized ShenMai saponins was based on calibration with their respective reference standards or calibration with a structurally similar reference standard.

Quantification of ShenMai ginsenosides in biological samples An AB Sciex API 4000 Q Trap mass spectrometer (Toronto, Canada), interfaced via a Turbo V ion source with an Agilent 1290 Infinity II LC (Waldbronn, Germany), was used for quantification of ShenMai ginsenosides in plasma, urine, and bile samples from the rat studies and in samples from the cell-based transport studies. Samples were prepared by precipitation with three volumes of methanol and follow-up centrifugation. Chromatographic separation was achieved on a $5 \mu \mathrm{m}$ Agilent ZORBAX Eclipse Plus $\mathrm{C}_{18}$ column $(50 \mathrm{~mm} \times 2.1 \mathrm{~mm}$ i.d.; Santa Clara, CA, USA) with a mobile phase, delivered at $0.35 \mathrm{~mL} / \mathrm{min}$, consisting of (A) methanol/water $(1: 99, \mathrm{v} / \mathrm{v}$; containing $25 \mu \mathrm{mol} / \mathrm{L}$ lithium acetate and $1 \mathrm{mmol} / \mathrm{L}$ formic acid) and (B) methanol/water (99:1, v/v; containing $25 \mu \mathrm{mol} /$ $\mathrm{L}$ lithium acetate and $1 \mathrm{mmol} / \mathrm{L}$ formic acid). A 12-min gradient program was used, consisting of $0-2 \mathrm{~min}$, from $20 \% \mathrm{~B}$ to $70 \% \mathrm{~B}$; 2-8 min, from $70 \% \mathrm{~B}$ to $85 \% \mathrm{~B} ; 8-8.5 \mathrm{~min}$, from $85 \% \mathrm{~B}$ to $100 \% \mathrm{~B}$; $8.5-10 \mathrm{~min}$, at $100 \% \mathrm{~B} ; 10-12 \mathrm{~min}$ at $20 \% \mathrm{~B}$. The mass spectrometer was operated in the positive ion mode to generate lithiated molecules. Optimal precursor-to-product ion pairs for multiple reaction monitoring of ginsenosides $R b_{1}, R b_{2} / R c, R d / R e, R a_{1} / R a_{2}$, $\mathrm{Rg}_{3}, \mathrm{Ra}_{3}, \mathrm{Rg}_{1}, \mathrm{Rg}_{2}, \mathrm{Rf}, \mathrm{Rh}_{1}, \mathrm{Ro}, \mathrm{Rg}_{6}, \mathrm{Rk}_{1} / \mathrm{Rg}_{5}$, and $\mathrm{Rh}_{4}$, notoginsenoside $R_{1}$, and 20-gluco-ginsenoside $R f$ were $\mathrm{m} / \mathrm{z}$ $1115 \rightarrow 349$ (collision energy, $81 \mathrm{~V}$ ), $1085 \rightarrow 319(85 \mathrm{~V}), 953 \rightarrow 773$ $(63 \mathrm{~V}), 1217 \rightarrow 451(83 \mathrm{~V}), 791 \rightarrow 349(67 \mathrm{~V}), 1247 \rightarrow 481(80 \mathrm{~V})$, $807 \rightarrow 627(51 \mathrm{~V}), 791 \rightarrow 187(77 \mathrm{~V}), 807 \rightarrow 349(63 \mathrm{~V}), 645 \rightarrow 465$ $(49 \mathrm{~V}), 963 \rightarrow 363$ (67 V), $773 \rightarrow 333(57 \mathrm{~V}), 773 \rightarrow 349$ (65 V), $627 \rightarrow$ $447(55 \mathrm{~V}), 939 \rightarrow 319(73 \mathrm{~V})$, and $969 \rightarrow 349(79 \mathrm{~V})$, respectively.

For quantification of saponins, matrix-matched calibration curves were constructed with reference standards using weighted $\left(1 / X\right.$ or $\left.1 / X^{2}\right)$ linear regression of the peak areas $(Y)$ of the analytes against the corresponding nominal analytes' concentrations ( $X$; $1.37,4.12,12.4,37.0,111,333,1000,3000$, and $5000 \mathrm{nmol} / \mathrm{L})$, and the curves showed good linearity $(R>0.99)$. Although no internal standard was used, assay validation, implemented according to the European Medicines Agency Guideline on bioanalytical method validation [38], demonstrated that the assays were reliable and reproducible for the intended use. The assays' lower limits of quantification were 1.37-37.0 $\mathrm{nmol} / \mathrm{L}$ for the test ginsenosides and the upper limits of quantification were 1000-5000 nmol/L. The intra-batch accuracy and precision were $85.2-113.9 \%$ and $1.5-15.0 \%$, respectively, while the inter-batch values were $85.3-113 \%$ and $2.1-14.9 \%$, respectively. The coefficients of variation of matrix factors were $2.5-14.3 \%$. The stability of analytes under conditions mimicking the analytical process was evaluated: after storage at $24^{\circ} \mathrm{C}$ for $5 \mathrm{~h}$, after storage at $8{ }^{\circ} \mathrm{C}$ for $24 \mathrm{~h}$ and after three freeze-and-thaw cycles. The test compounds were stable under the test conditions, because the measured mean concentrations fluctuated within $-0.2-14.6 \%$ of the nominal concentrations. All the preceding validation results were within the required ranges.

Liquid chromatography/mass spectrometry-based bioanalytical assay for quantification of $E_{2} 17 \beta G$ was the same as that described by Jiang et al. [14].

\section{Data processing}

ShenMai contains many saponins and their daily doses from the injection are a governing factor for their individual contributions in potential ShenMai-drug interactions. Therefore, the detected and characterized ShenMai saponins were ranked according to their daily doses, which were calculated as the products of their content levels in the injection and the injection's label daily dose $(100 \mathrm{~mL} /$ day). Then, these saponins were graded into different levels according to their daily doses, i.e., $>10,1-10$, and $<1 \mu \mathrm{mol} /$ day. Due to differences in formulation and dosage regimen among herbal medicines, this ranking and grading is based on the compounds' daily doses, rather than on their content levels in the herbal medicine; this facilitates data comparison among such medicines.

Pharmacokinetic parameters of circulating ShenMai saponins were estimated by non-compartmental analysis using Innaphase Kinetica software package (version 5.0; Philadelphia, PA, USA). Fractions of dose excreted into urine $\left(f_{\mathrm{e}-\mathrm{u}}\right)$ and into bile $\left(f_{\mathrm{e}-\mathrm{B}}\right)$ were calculated using the cumulative amounts excreted into urine $\left(\right.$ Cum. $\left.A_{\mathrm{e}-\mathrm{u}}\right)$ and into bile $\left(\right.$ Cum. $\left.A_{\mathrm{e}-\mathrm{B}}\right)$, respectively, divided by the dose. $\mathrm{A}$ ratio of renal clearance to the product of glomerular filtration rate and unbound fraction in plasma $\left[\mathrm{CL}_{R} /\right.$ $\left.\left(G F R \times f_{\text {u-plasma }}\right)\right]$ was used to understand which process(es) were involved in the renal excretion. The hepatobiliary $\left(C_{B}\right)$ and renal clearance $\left(C_{R}\right)$ were calculated from the cumulative amounts excreted into bile and into urine divided by the area under plasma concentration-time curve, i.e., Cum. $A_{\mathrm{e}-\mathrm{B}} / \mathrm{AUC}_{0-\mathrm{t}}$ and $C u m . A_{\mathrm{e}-\mathrm{U}} /$ $\mathrm{AUC}_{0-\mathrm{t}}$, respectively.

In vitro $\mathrm{IC}_{50}$ was estimated using Erithacus GraFit software (version 5.0; Surrey, UK). Contributions of individual circulating ginsenosides to OATP1B-mediated ShenMai-drug interaction potential were estimated based on their $\left(C \times f_{\text {u-plasma }}\right) / / C_{50}$ ratios, after a single dose of ShenMai, and on their $\left(C \times R \times f_{\text {u-plasma }}\right) / / C_{50}$ ratios, at steady state after multiple doses of ShenMai. The term $C$ is the rat plasma concentration of the ginsenoside after starting a 
30-min intravenous infusion of ShenMai or after starting such infusion on the first day of multiple dosing; the term $R$ is the accumulative factor of the ginsenoside calculated as $1 /\left(1-e^{-k \tau}\right)$, where $k$ is its elimination rate constant $\left(0.693 / t_{1 / 2}\right)$ and $\tau$ is the dosing interval $(24 \mathrm{~h})$. The term $f_{\mathrm{u} \text {-plasma }}$ is unbound fraction of the ginsenoside in human plasma. Given uncertainties in measurements of binding in plasma, the $f_{\text {u-plasma }}$ was set to $1 \%$ when experimentally determined to be $<1 \%$ [39]. The term $I C_{50}$ of the ginsenoside is for the human OATP1B3 or OATP1B1. All results are expressed as arithmetic mean \pm standard deviation.

\section{RESULTS}

Saponins detected in ShenMai and their relative abundance A total of 49 saponins were detected and characterized in samples of the three ShenMai lots (Table 1 and Fig. 1), i.e., 47 ginsenosides originating from the component herb Hongshen (steamed $P$. ginseng roots) [20 ppd-type ginsenosides (1-20), 13 ppt-type ginsenosides (31-43), and 14 ginsenosides of other types (51-64)] and two ophiopogonins (71 and 72) originating from the other component herb Maidong (O. japonicus roots). Based on their individual doses from ShenMai, these saponins were graded into three levels: 10-100 $\mu \mathrm{mol} /$ day [the ppd-type ginsenosides $\mathrm{Rb}_{1}(\mathbf{1})$, $\mathrm{Rb}_{2}(\mathbf{2})$, and $\mathrm{Rc}(\mathbf{3})$, the ppt-type ginsenoside $\mathrm{Rg}_{1}(\mathbf{3 1})$ and $\mathrm{Re}(\mathbf{3 2})$, and the other-type ginsenoside Ro (51)], 1-10 $\mu \mathrm{mol} /$ day [the ppdtype ginsenosides $R d(4), R_{1}(5), R_{3}(6), R_{2}(\mathbf{7}), R_{3}(\mathbf{8})$, and $R b_{3}$ (9), the ppt-type ginsenoside $\operatorname{Rg}_{2}(33), R f(34)$, notoginsenoside $R_{1}$ (35), and 20-gluco-ginsenoside $\mathrm{Rf}$ (36), and the other-type ginsenosides $\mathrm{Rg}_{6}(\mathbf{5 2}), \mathrm{Rk}_{1}(\mathbf{5 3}), \mathrm{Rh}_{4}(\mathbf{5 4})$, and $\left.\mathrm{Rg}_{5}(\mathbf{5 5})\right]$, and $<1$ $\mu \mathrm{mol} /$ day [the remaining 27 minor ginsenosides (10-20, 37-43, and 56-64) and ophiopogonin $D(71)$ and $D^{\prime}(72)$ ]. The sum of doses of Level I saponins was $62 \%$ of the total dose of saponins in ShenMai while the sum of doses of Level II saponins was $33.4 \%$ of the total dose of saponins. The remaining minor saponins constituted only $4.6 \%$ of the total dose of saponins. The injection exhibited lot-to-lot variability of $6.9-18.4 \%$ for the Level I saponins, $2.2-18.1 \%$ for the Level II saponins and $3.4-28.2 \%$ for the remaining saponins. Although flavonoids were also reported to be present in Maidong [34], they were not detected in the test ShenMai samples.

\section{Systemic exposure to and pharmacokinetics of saponins in rats} that received ShenMai

A total of 20 ginsenosides circulating during and after a $30-\mathrm{min}$ intravenous infusion of ShenMai $(10 \mathrm{~mL} / \mathrm{kg})$ were detected in rats and characterized. These consisted of eight ppd-type ginsenosides, i.e., ginsenosides $\mathrm{Rb}_{1}(\mathbf{1}), \mathrm{Rb}_{2}(\mathbf{2}), \mathrm{Rc}(\mathbf{3}), \operatorname{Rd}(\mathbf{4}), \mathrm{Ra}_{1}(\mathbf{5}), \mathrm{Ra}_{2}$ (7), $\mathrm{Ra}_{3}(\mathbf{8})$, and $\mathrm{Rg}_{3}(\mathbf{6})$, in descending order of exposure levels $\left(C_{\text {max }}\right)$; seven ppt-type ginsenosides, i.e., ginsenosides $\mathrm{Rg}_{1}(\mathbf{3 1})$, $\mathrm{Re}$ (32), $\mathrm{Rg}_{2}$ (33), and $\mathrm{Rf}(\mathbf{3 4})$, notoginsenoside $\mathrm{R}_{1}$ (35), 20-glucoginsenoside $\mathrm{Rf}(\mathbf{3 6})$, and ginsenoside $\mathrm{Rh}_{1}$ (37); and five ginsenosides of other types, i.e., ginsenosides $\mathrm{Ro}(\mathbf{5 1}), \mathrm{Rg}_{6}(\mathbf{5 2}), \mathrm{Rk}_{1}(\mathbf{5 3})$, $\mathrm{Rh}_{4}$ (54), and $\mathrm{Rg}_{5}$ (55). The minor Hongshen ginsenosides and Maidong ophiopogonins (dose, $<1 \mu \mathrm{mol} /$ day) were negligibly or not detected in plasma after dosing. Oxidized metabolites of the circulating ShenMai ginsenosides [except for ginsenosides $\mathrm{Rg}_{3}(\mathbf{6})$, $\mathrm{Rg}_{2}$ (33), $\mathrm{Rf}(\mathbf{3 4})$, and $\left.\mathrm{Rh}_{1}(\mathbf{3 7})\right]$ and the deglycosylated metabolites compound-K, 20(S)-protopanaxadiol, or 20(S)-protopanaxatriol were negligibly or not detected. Although the oxidized metabolites of ginsenosides $\mathrm{Rg}_{3}(\mathbf{6}), \mathrm{Rg}_{2}(\mathbf{3 3}), \mathrm{Rf}(\mathbf{3 4})$, and $\mathrm{Rh}_{1}$ (37) were detected in plasma after dosing ShenMai, their levels were low compared with those of the respective parent compounds. These metabolites were mainly eliminated via hepatobiliary excretion. Chemical structures of the major circulating ginsenosides (1-8, 31-37, and 51-55) and those of ophiopogonins $D(71)$ and $D^{\prime}(\mathbf{7 2})$ are shown in Fig. 2.

Table 2 summarizes the pharmacokinetics of circulating ginsenosides (1-8, 31-37, and 51-55) from intravenously dosed
ShenMai; Fig. 3a-d shows the total (unbound plus bound) plasma concentrations of these ginsenosides over time. Significant differences in total levels and duration of systemic exposure were observed among different types of ginsenosides. Due to significant inter-compound differences in content level in ShenMai, comparison of total levels of systemic exposure among the ginsenosides should be based on correction of these data by individual ginsenoside doses from the injection. As a result, the ppd-type ginsenosides (1-8) exhibited substantially higher total levels of systemic exposure than the ppt-type ginsenosides (3137) and ginsenosides of other types (51-55). For the ppd-type ginsenosides, $C_{30 \mathrm{~min}} /$ compound dose and AUC/compound dose were $1.38-2.23 \mu \mathrm{mol} / \mathrm{L} / \mu \mathrm{mol}$ and $8.97-26.14 \mu \mathrm{mol} / \mathrm{L} \mathrm{h} / \mu \mathrm{mol}$, respectively (except for ginsenoside $\mathrm{Rg}_{3}$ (6), $0.66 \mu \mathrm{mol} / \mathrm{L} / \mu \mathrm{mol}$ and $0.50 \mu \mathrm{mol} / \mathrm{L} \mathrm{h} / \mu \mathrm{mol}$, respectively), while for the ppt-type/ other types, such data were $0.08-0.31 / 0.12-0.73 \mu \mathrm{mol} / \mathrm{L} / \mu \mathrm{mol}$ and $0.04-0.23 / 0.07-0.59 \mu \mathrm{mol} / \mathrm{L} \mathrm{h} / \mu \mathrm{mol}$, respectively. Plasma protein binding also differed significantly among these ginsenosides (Table 3). The ppd-type ginsenosides (1-8) were extensively bound in plasma, while the ppt-type ginsenosides (31-37) were not extensively bound. Regarding ginsenosides of other types, ginsenoside Ro (51) exhibited a $f_{\mathrm{u} \text {-plasma }}$ of $22 \%$, while such values for ginsenosides $\mathrm{Rk}_{1}(\mathbf{5 3}) / \mathrm{Rg}_{5}(\mathbf{5 5})$ and ginsenosides $\mathrm{Rg} 6{ }_{6}(\mathbf{5 2}) / \mathrm{Rh}_{4}$ (54) were $<0.1 \%$ and around $4 \%$, respectively. Based on the products of total exposure levels and $f_{\text {u-plasma }}$ values, levels of systemic exposure to unbound ginsenosides over time are shown in Fig. $3 \mathrm{e}-\mathrm{h}$. It is worth mentioning that such $f_{\text {u-plasma }}$ values for humans were greater than the respective values for rats (Table 3 ); these interspecies differences should be considered in predicting the related drug interaction in humans. In addition, terminal halflife $\left(t_{1 / 2}\right)$ also differed significantly among the ginsenosides (Table 2). The terminal half-lives of the ppd-type ginsenosides (1-8) [except for ginsenoside $\mathrm{Rg}_{3}(\mathbf{6})$ ] were substantially longer than those of the ppt-type ginsenosides (31-37) and the ginsenosides of other types (51-55).

Systemic clearance $\left(\mathrm{CL}_{\text {tot,p }}\right)$ differed notably among the different types of circulating ginsenosides (Table 2). The $\mathrm{CL}_{\text {tot,p }}$ values of the ppd-type ginsenosides (1-5, 7, and 8), except for ginsenoside $\mathrm{Rg}_{3}$ (6), were significantly lower than those of the ppt-type ginsenosides (31-37) and the ginsenosides of other types (51-55). However, the apparent volumes of distribution at steady state $\left(V_{\mathrm{sS}}\right)$ did not differ so much among these ginsenosides (Table 2 ). Clearly, the inter-ginsenoside differences in systemic exposure correlated mainly with the differences in $\mathrm{CL}_{\text {tot,p }}$, rather than those in $V_{\text {SS. }}$.

\section{Elimination of ginsenosides in rats that received ShenMai} For the ppd-type ginsenosides (1-8), renal and hepatobiliary excretion of the unchanged compounds represented their major routes of elimination (Table 2). These ppd-type ginsenosides, except ginsenosides $\mathrm{Rd}(4)$ and $\mathrm{Rg}_{3}(\mathbf{6})$, exhibited greater $f_{\mathrm{e}-\mathrm{U}, 0-72 \mathrm{~h}}$ values than the respective $f_{\mathrm{e}-\mathrm{B}, 0-72 \mathrm{~h}}$ values. Their renal excretion appeared to be mostly based on glomerular filtration as indicated by their $\mathrm{CL}_{R} /\left(\mathrm{GFR} \times f_{\mathrm{u} \text {-plasma }}\right)$ values. Ginsenoside $\mathrm{Rd}$ (4) exhibited an extraordinarily high $f_{\mathrm{e}-\mathrm{B}, 0-72 \mathrm{~h}}$ value $(91.4 \%)$, the sum of which with $f_{\mathrm{e}-\mathrm{U}, 0-72 \mathrm{~h}}$ exceeded $100 \%$ (i.e., $109.1 \%$ ), suggesting that part of this circulating ginsenoside derived from metabolism of other ShenMai ginsenosides. Ginsenoside $\mathrm{Rg}_{3}$ (6) was eliminated mainly by hepatobiliary excretion; its renal excretion appeared to be negligible.

For the ppt-type ginsenosides (31-37), renal and hepatobiliary excretion of the unchanged compounds contributed comparably to their elimination (Table 2). Consistent with the inter-compound differences in $\mathrm{CL}_{\text {tot,p }}$, the hepatobiliary excretory clearance $\left(\mathrm{CL}_{\mathrm{B}}\right)$ and renal excretory clearance $\left(\mathrm{CL}_{\mathrm{R}}\right)$ values for these ppt-type ginsenosides were significantly greater than such respective values for the ppd-type ginsenosides (1-8). The sums of $f_{\mathrm{e}-}$ $\mathrm{B}, 0-72 \mathrm{~h}$ and $f_{\mathrm{e}-\mathrm{U}, 0-72 \mathrm{~h}}$ values for ginsenosides $\mathrm{Rg}_{2}(\mathbf{3 3}), \mathrm{Rf}(\mathbf{3 4})$, and 


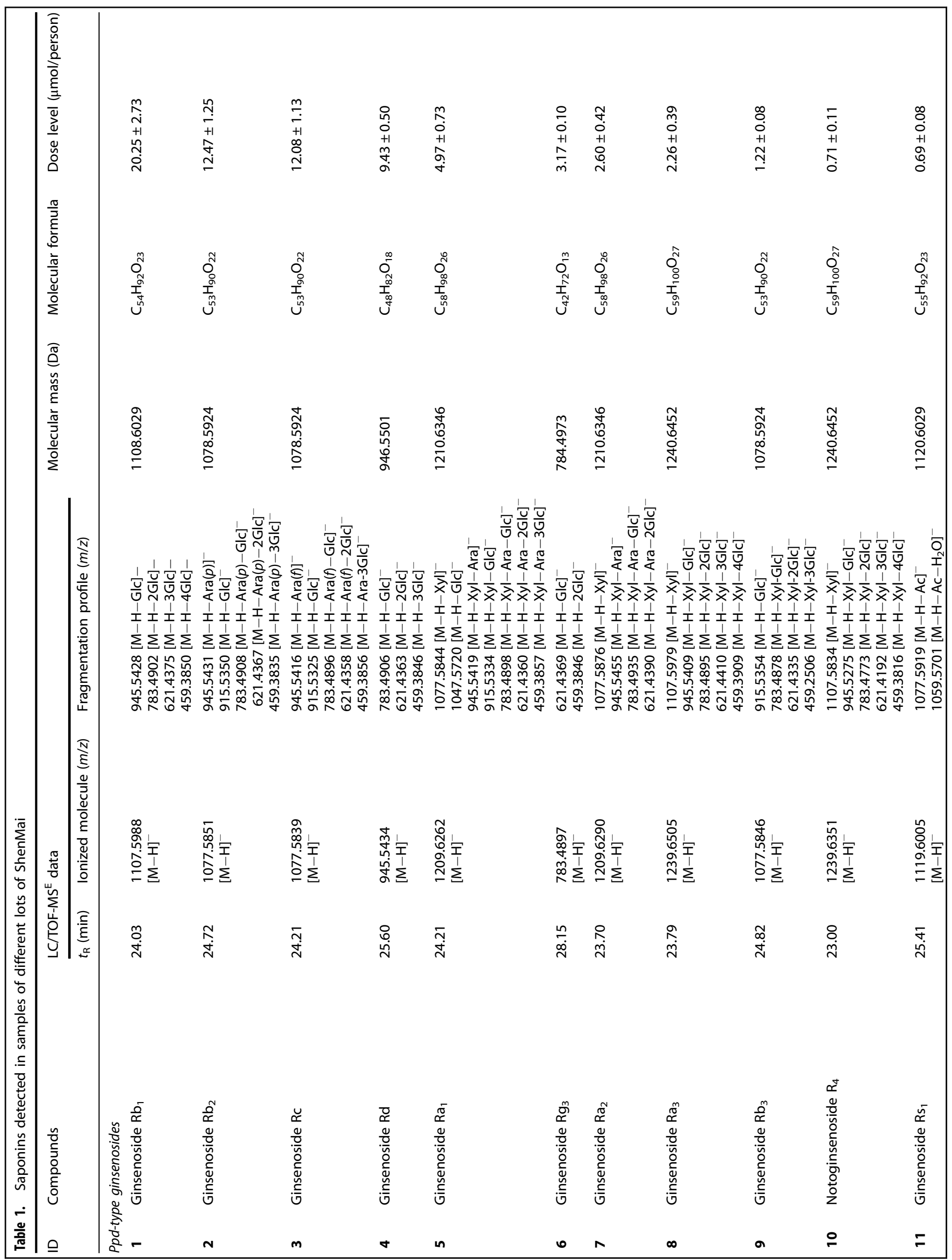




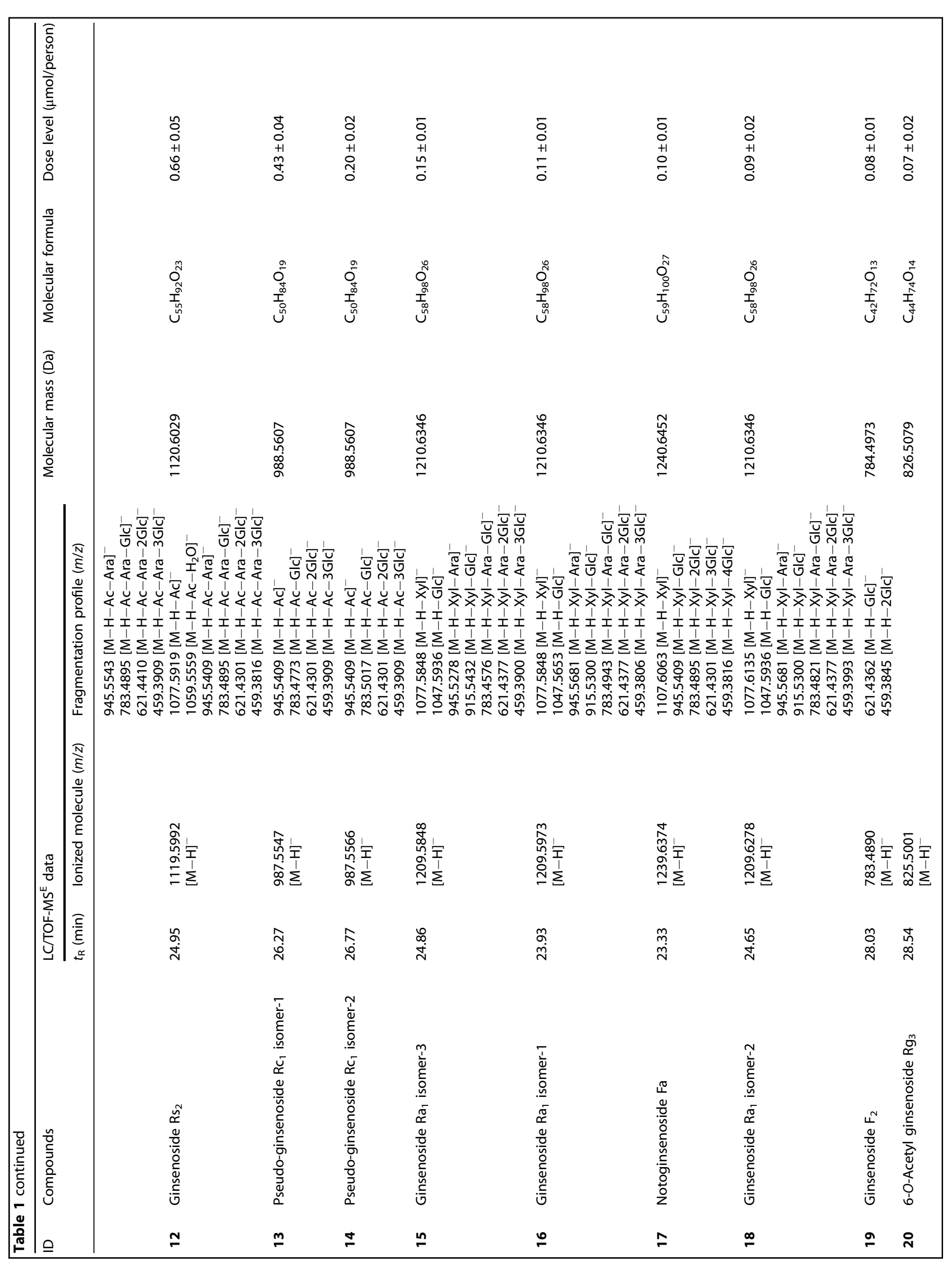




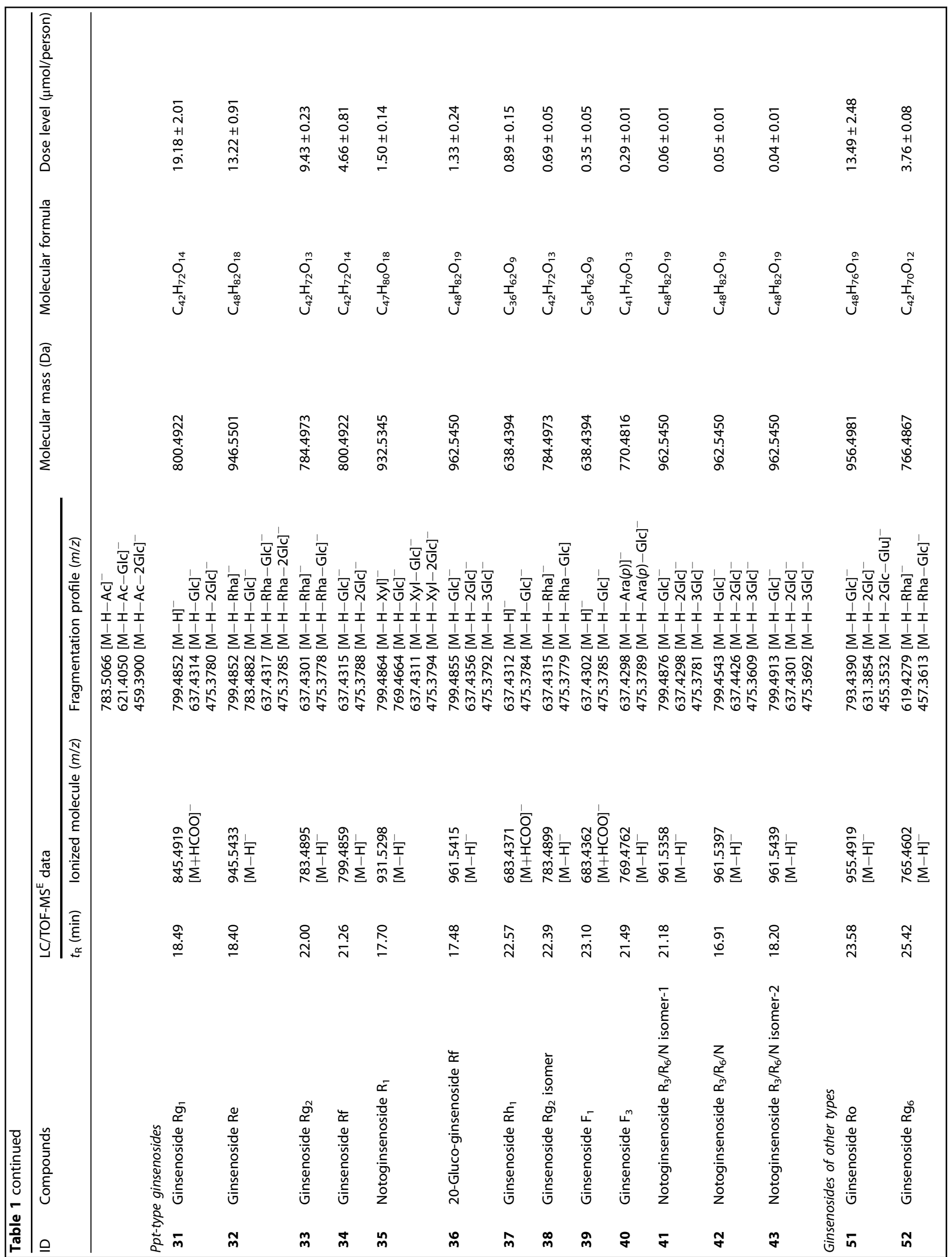




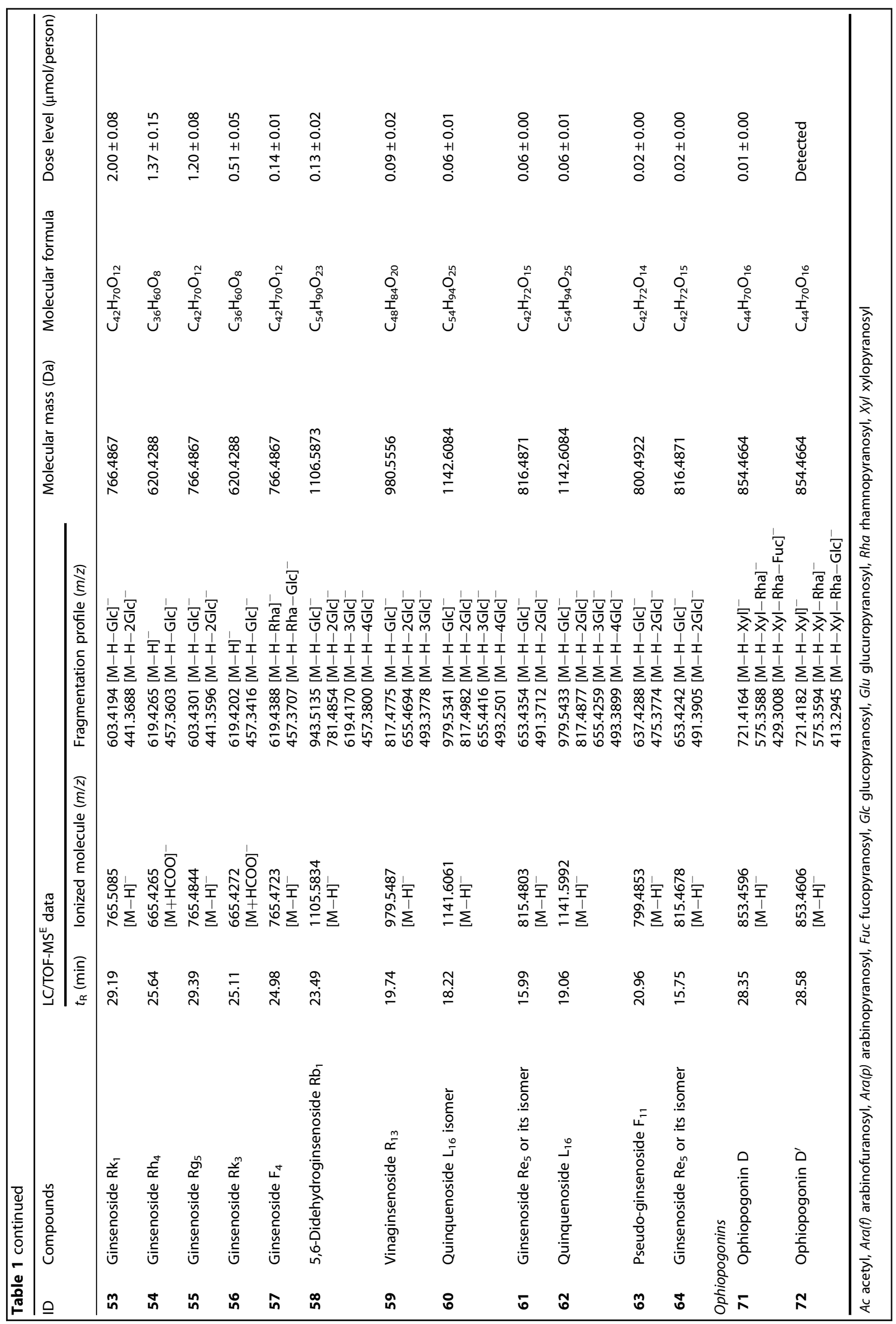



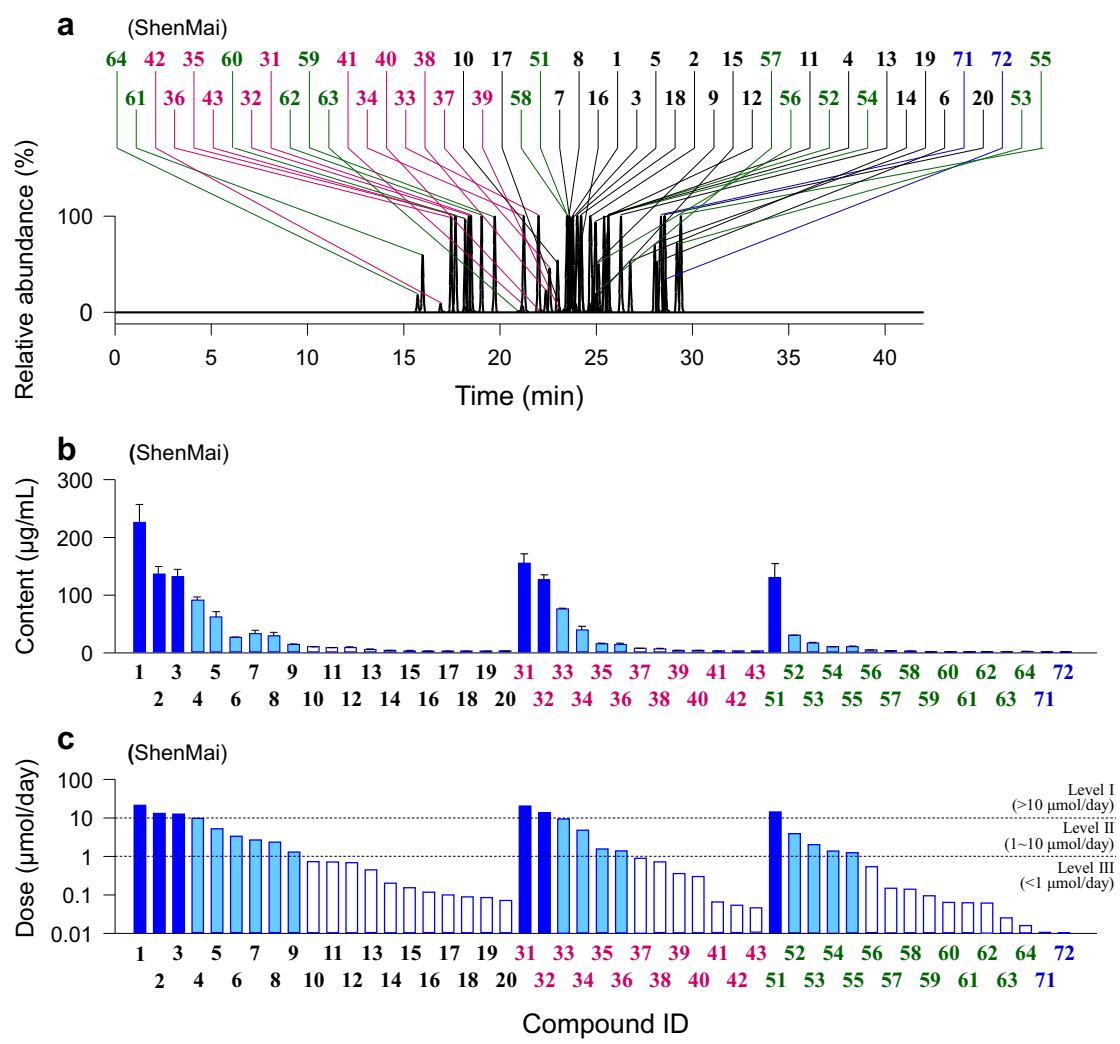

Fig. 1 Saponins, detected by mass spectrometry, in ShenMai. a Stacked chromatograms of saponins in a typical sample of ShenMai. b Mean content levels of saponins in three lots of ShenMai. c Mean doses of saponins from the three lots of ShenMai at the label dose $100 \mathrm{~mL} / \mathrm{day}$
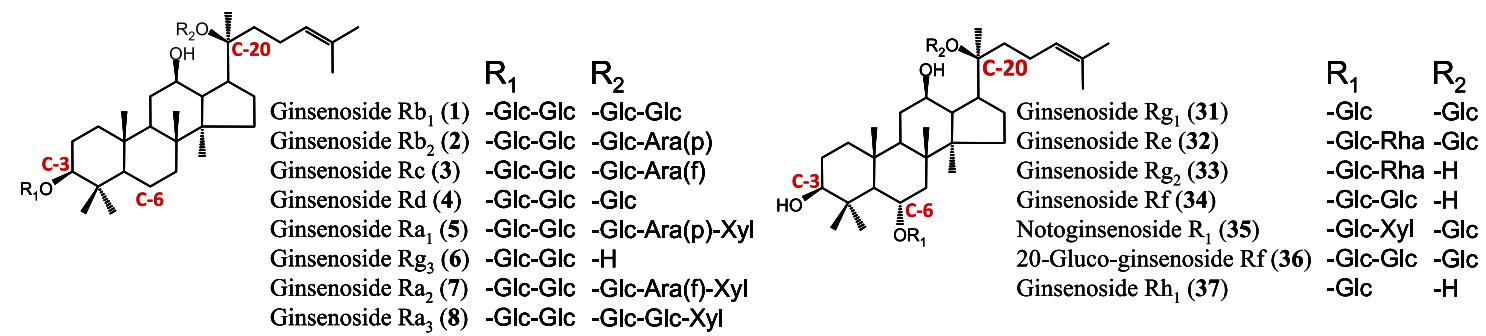

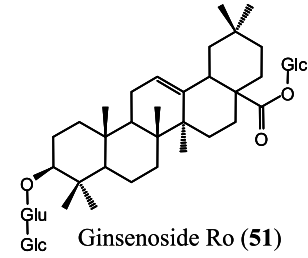

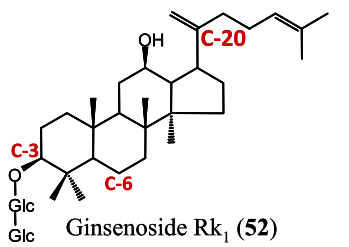

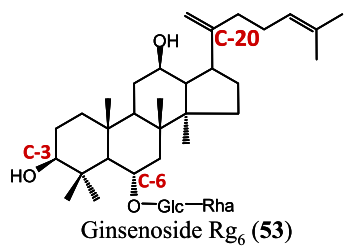

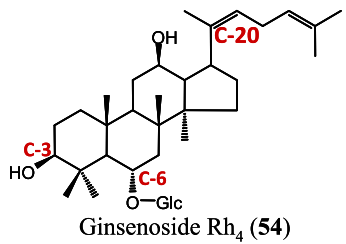

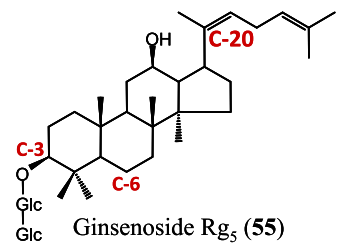

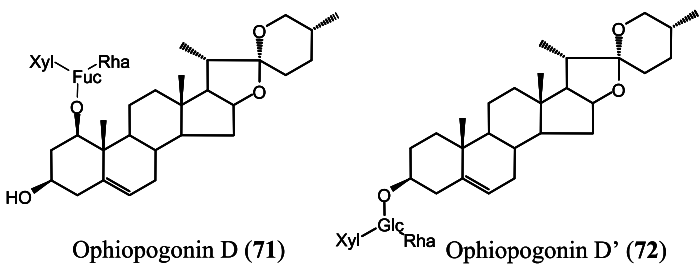

Fig. 2 Chemical structures of major circulating ginsenosides in rats that received an intravenous infusion of ShenMai and those of major ophiopogonins present in the herbal injection. Ara(p) arabinopyranosyl, Ara(f) arabinofuranosyl, Fuc fucopyranosyl, Glc glucopyranosyl, Glu glucuropyranosyl, Rha rhamnopyranosyl, Xyl xylopyranosyl 


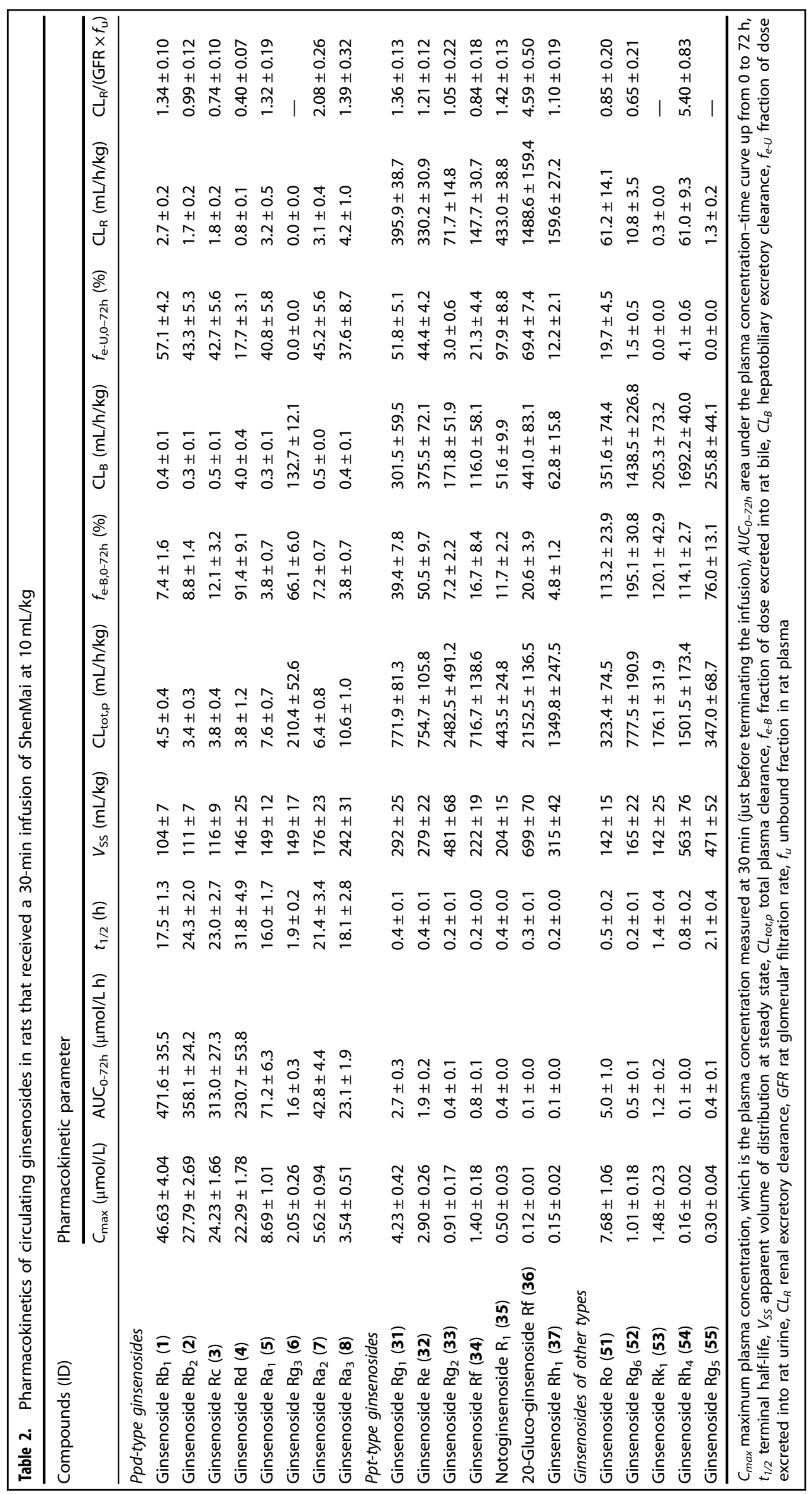



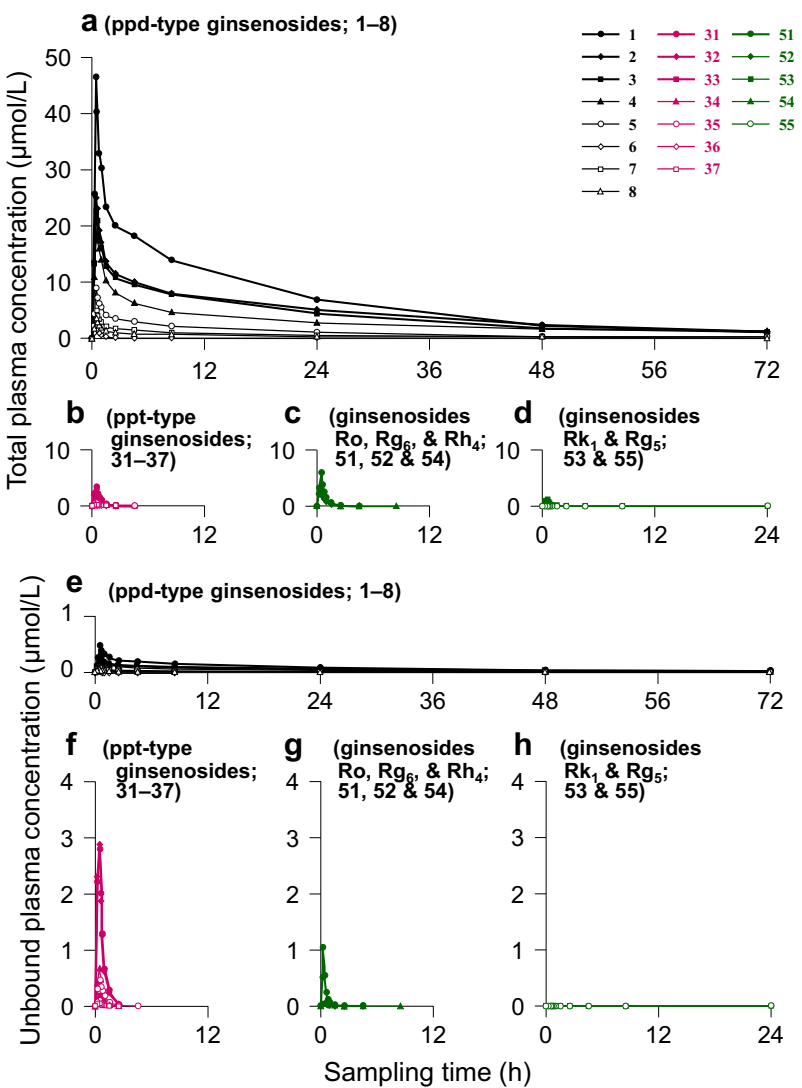

Fig. 3 Plasma concentrations of various ginsenosides over time after a 30-min intravenous infusion of ShenMai in rats. a-d Total (unbound plus bound) concentrations of ginsenosides in rat plasma over time. $\mathbf{e}-\mathbf{h}$ Unbound concentrations of ginsenosides in rat plasma over time. The total plasma concentrations of ophiopogonins $D(\mathbf{7 1})$ and $D^{\prime}(\mathbf{7 2})$ were too low to be quantified in rat plasma after dosing ShenMai. Given uncertainties in measurements of binding in plasma, the $f_{\mathrm{u} \text {-plasma }}$ is set to $1 \%$ when experimentally determined to be $<1 \%$

$\mathrm{Rh}_{1}$ (37) were small (10-38\%). For the ginsenosides of other types (51-55), hepatobiliary excretion contributed the main part of their elimination, while their renal excretion was negligible. Renal excretion of the ppt-type ginsenosides [31-37, except 20-glucoginsenoside $\mathrm{Rf}(\mathbf{3 6})]$ and ginsenosides of other types [51-55, except ginsenoside $\mathrm{Rh}_{4}(\mathbf{5 4})$ ] appeared to be mainly based on glomerular filtration, as indicated by their $\mathrm{CL}_{\mathrm{R}} /\left(\mathrm{GFR} \times f_{\mathrm{u} \text {-plasma }}\right)$ values (Table 2 ).

In vitro metabolism of circulating ShenMai ginsenosides As shown in Fig. 4, incubation of ginsenoside $\mathrm{Rb}_{1}(\mathbf{1})$ with rat liver homogenate resulted in significant formation of ginsenoside $\mathrm{Rd}$ (4). However, such incubation of ginsenosides $\mathrm{Rb}_{2}(\mathbf{2})$ and $\mathrm{Ra}_{3}(\mathbf{8})$ yielded only low and trace amount of ginsenoside $\mathrm{Rd}(\mathbf{4})$; such incubation of ginsenosides $R c(3), \operatorname{Ra}_{1}(5)$, and $\mathrm{Ra}_{2}$ (7) resulted in negligible formation of ginsenoside $\mathrm{Rd}(\mathbf{4})$. The in vitro deglycosylation, which is expected to take place in vivo, might be the reason, at least in part, for the extraordinarily high $f_{\mathrm{e}-\mathrm{B}, 0-72 \mathrm{~h}}$ of ginsenoside $\mathrm{Rd}(\mathbf{4})$ in rats (Table 2 ). Similar inter-compound differences in formation of ginsenoside Rd (4) were observed by incubation of these ppd-type ginsenosides with human liver cytosol (Fig. 4). It is worth mentioning that rat liver cytosol was also tested for the metabolism above. However, unlike using human liver cytosol, no formation of ginsenoside Rd (4) was observed using rat liver cytosol. Despite using different liver
Table 3. Unbound fractions of ShenMai ginsenosides in rat and human plasma

\begin{tabular}{|c|c|c|}
\hline \multirow[t]{2}{*}{ Compounds (ID) } & \multicolumn{2}{|l|}{$f_{\text {u-plasma }}(\%)$} \\
\hline & Rat & Human \\
\hline \multicolumn{3}{|l|}{ Ppd-type ginsenosides } \\
\hline Ginsenoside $\mathrm{Rb}_{1}(\mathbf{1})$ & $0.62 \pm 0.03$ & $1.7 \pm 0.6$ \\
\hline Ginsenoside $\mathrm{Rb}_{2}(\mathbf{2})$ & $0.51 \pm 0.08$ & $2.4 \pm 1.1$ \\
\hline Ginsenoside Rc (3) & $0.74 \pm 0.33$ & $1.5 \pm 0.1$ \\
\hline Ginsenoside Rd (4) & $0.59 \pm 0.01$ & $1.2 \pm 0.2$ \\
\hline Ginsenoside $\mathrm{Ra}_{1}(\mathbf{5})$ & $0.74 \pm 0.01$ & $2.0 \pm 0.1$ \\
\hline Ginsenoside $\mathrm{Rg}_{3}(\mathbf{6})$ & $1.0 \pm 0.1$ & $0.67 \pm 0.17$ \\
\hline Ginsenoside $\mathrm{Ra}_{2}$ (7) & $0.46 \pm 0.03$ & $1.7 \pm 0.4$ \\
\hline Ginsenoside $\mathrm{Ra}_{3}(\mathbf{8})$ & $0.92 \pm 0.13$ & $4.0 \pm 0.7$ \\
\hline \multicolumn{3}{|l|}{ Ppt-type ginsenosides } \\
\hline Ginsenoside $\mathrm{Rg}_{1}$ (31) & $89.0 \pm 0.7$ & $98.5 \pm 5.3$ \\
\hline Ginsenoside Re (32) & $83.4 \pm 3.2$ & $94.8 \pm 7.6$ \\
\hline Ginsenoside $\mathrm{Rg}_{2}$ (33) & $20.8 \pm 0.5$ & $38.7 \pm 4.6$ \\
\hline Ginsenoside Rf (34) & $53.5 \pm 5.0$ & $50.6 \pm 4.6$ \\
\hline Notoginsenoside $\mathrm{R}_{1}$ (35) & $93.4 \pm 0.3$ & $97.3 \pm 2.5$ \\
\hline 20-Gluco-ginsenoside Rf (36) & $99.2 \pm 6.6$ & $105.7 \pm 4.2$ \\
\hline Ginsenoside $\mathrm{Rh}_{1}(\mathbf{3 7})$ & $44.4 \pm 0.4$ & $34.6 \pm 0.9$ \\
\hline \multicolumn{3}{|l|}{ Ginsenosides of other types } \\
\hline Ginsenoside Ro (51) & $22.1 \pm 2.4$ & $14.6 \pm 2.2$ \\
\hline Ginsenoside $\mathrm{Rg}_{6}$ (52) & $5.1 \pm 0.5$ & $4.6 \pm 0.3$ \\
\hline Ginsenoside $\mathrm{Rk}_{1}$ (53) & $0.04 \pm 0.00$ & $0.02 \pm 0.00$ \\
\hline Ginsenoside $\mathrm{Rh}_{4}(\mathbf{5 4})$ & $3.5 \pm 0.5$ & $4.3 \pm 0.8$ \\
\hline Ginsenoside $\mathrm{Rg}_{5}(\mathbf{5 5})$ & $0.07 \pm 0.00$ & $0.09 \pm 0.02$ \\
\hline
\end{tabular}

preparations for the two species, the overall result essentially indicates that the deglycosylation can take place in the rat liver and also in human liver.

Mono-oxidized metabolites of ginsenosides $\mathrm{Rg}_{3}(6), \mathrm{Rg}_{2}(33), \mathrm{Rf}$ (34), and $\mathrm{Rh}_{1}$ (37) were detected after the compounds were incubated with NADPH-fortified rat liver microsomes (Fig. 4). This might explain, at least in part, why these ginsenosides had low sums of $f_{\mathrm{e}-\mathrm{B}, 0-72 \mathrm{~h}}$ and $f_{\mathrm{e}-\mathrm{U}, 0-72 \mathrm{~h}}$ after dosing ShenMai in rats (Table 2). Similar in vitro metabolism was observed for these ginsenosides by incubating with $\mathrm{NADPH}$-fortified human liver microsomes (Fig. 4).

In vitro interactions of circulating ShenMai ginsenosides and ophiopogonins with rat Oatp1b2, human OATP1B1, and human OATP1B3

As shown in Table 4, most test ShenMai saponins exhibited slow rates of membrane permeation $(<10 \mathrm{pmol} / \mathrm{min} / \mathrm{mg}$ protein) in mock cells, except for ginsenosides $\mathrm{Rg}_{5}(\mathbf{5 5}), \mathrm{Rk}_{1}(\mathbf{5 3})$, and $\mathrm{Rh}_{4}(\mathbf{5 4})$ and ophiopogonin $\mathrm{D}^{\prime}(\mathbf{7 2})$ with a rapid rate of membrane permeation ( $>400 \mathrm{pmol} / \mathrm{min} / \mathrm{mg}$ protein) and ginsenoside $\mathrm{Rg}_{3}$ (6) and ophiopogonin $D$ (71) with a moderately rapid rate (around $100 \mathrm{pmol} / \mathrm{min} / \mathrm{mg}$ protein). Among the test ginsenosides with a slow rate of membrane permeation, most of the ppttype ginsenosides $(\mathbf{3 1}, \mathbf{3 2}$, and 34-37) were substrates of rat Oatp1 $1 \mathrm{~b} 2$ and selective substrates of human OATP1B3, based on their net transport ratios (Table 4). Ginsenoside $\mathrm{Rg}_{2}$ (33; ppt-type) and ginsenosides $\mathrm{Ro}(\mathbf{5 1})$ and $\mathrm{Rg}_{6}(\mathbf{5 2})$ of other types were substrates of both human OATP1B1 and OATP1B3, as well as Oatp1b2. Cellular uptakes of these test ginsenosides $(\mathbf{3 1 - 3 7 , 5 1}$, 

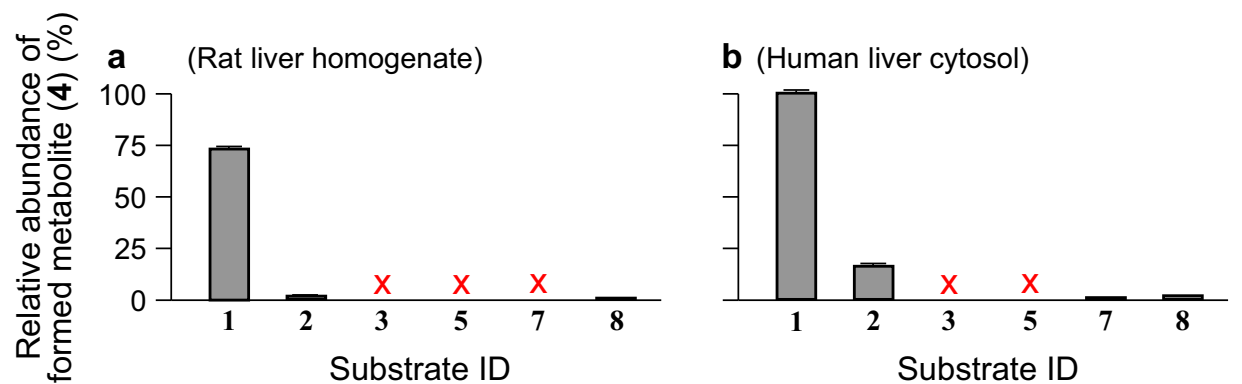

C (Rat liver microsomes)
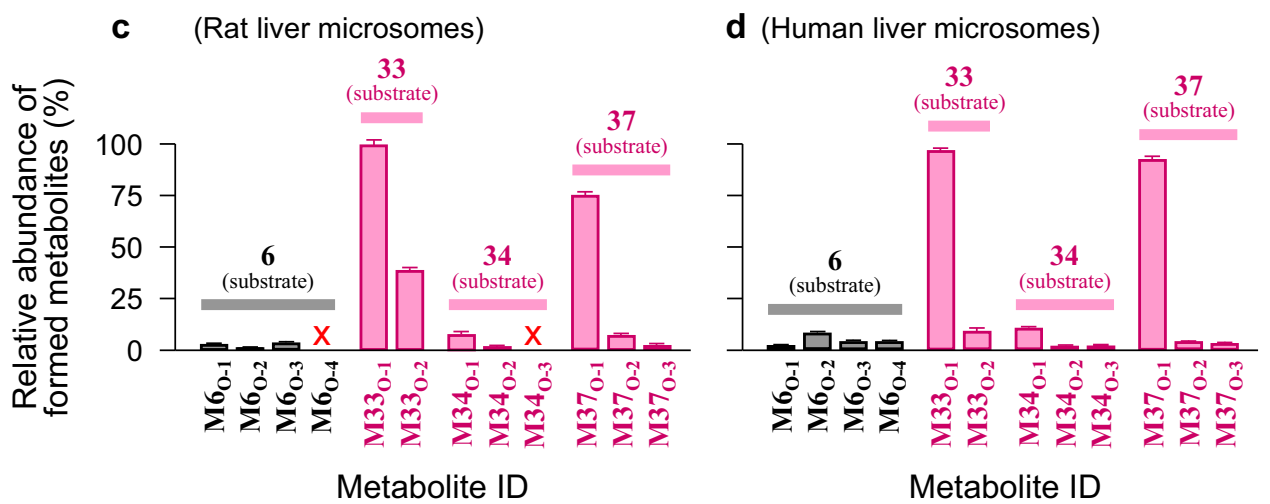

Fig. 4 In vitro deglycosylation of the ppd-type ginsenosides $R b_{1}(\mathbf{1}), R b_{2}(\mathbf{2}), R c(3), R_{1}(\mathbf{5}), R_{2}(\mathbf{7})$, and $R a_{3}(\mathbf{8})$ into the metabolite ginsenoside $\mathrm{Rd}$ (4) using rat liver homogenate (a) compared with that using human liver cytosol (b) and in vitro oxidation of ginsenosides $\mathrm{Rg}_{3}(\mathbf{6}), \mathrm{Rg}_{2}(\mathbf{3 3})$, $\mathrm{Rf}$ (34), and $\mathrm{Rh}_{1}$ (37) using NADPH-fortified rat liver microsomes (c) compared with that using NADPH-fortified human liver microsomes (d). Metabolite ID provides information regarding parent compound, metabolite type, and metabolite isomer. For instance, M6 in $\mathbf{M} \mathbf{6}_{\mathbf{O}-\mathbf{1}}$ denotes a metabolite of ginsenosides $\mathrm{Rg}_{3}(\mathbf{6})$, the subscript letter $\mathrm{O}$ indicating that it is an oxidized metabolite and the subscript number 1 indicating it is the metabolite's first eluted isomer. Structural similarities and differences of these ginsenosides are shown in Fig. 2

and $\mathbf{5 2}$ ) by the transporters were inhibited by $100 \mu \mathrm{mol} / \mathrm{L}$ rifampin, all with a reduced net transport ratio $<3$. OATP1B1 and OATP1B3 had limited influence on cellular transport of the ppt-type ginsenoside $\mathrm{Rh}_{1}(\mathbf{3 7})$, based on their net transport ratios for this compound. Meanwhile, the ppd-type ginsenosides (1-8) were not substrates of Oatp1b2, OATP1B1, or OATP1B3. Regarding the test saponins with a rapid or not-so-rapid rate of membrane permeation, their net transport ratios for Oatp1b2, OATP1B1, and OATP1B3 were all $<3$, except for ophiopogonin $D$ (71) with net transport ratios of around 4 for OATP1B1 and OATP1B3.

As shown in Table 5 , most of the test ginsenosides at $100 \mu \mathrm{mol} / \mathrm{L}$ exhibited $>50 \%$ inhibition of OATP1B1- and OATP1B3-mediated cellular uptake of $E_{2} 17 \beta G$, except for notoginsenoside $R_{1}$ (35) and 20-glucoginsenoside $\mathrm{Rf}$ (36) exhibiting such inhibition for OATP1B3 only. These ginsenosides more potently inhibited OATP1B3 than OATP1B1 $\left(\right.$ IC $_{50}$ values shown in Table 5; associated dose-response curves shown in Supplementary Fig. S1 and S2). Ppd-type ginsenosides (1-8) exhibited potent OATP1B3 inhibition with $\mathrm{IC}_{50}$ of $<3 \mu \mathrm{mol} / \mathrm{L}$, and ginsenoside $\mathrm{Rg}_{3}$ (6) was the most potent inhibitor. Ginsenosides of other types (51-55) also potently inhibited OATP1B3 (Table 5). In contrast, most of the ppt-type ginsenosides (31, 32, and 34-36) exhibited $\mathrm{IC}_{50}$ values of $>10 \mu \mathrm{mol} / \mathrm{L}$ for OATP1B3; such values for ginsenosides $\mathrm{Rg}_{2}$ (33) and $\mathrm{Rh}_{1}$ (37) were around $3 \mu \mathrm{mol} / \mathrm{L}$. Although some known inhibitors of OATP1B1 and OATP1B3 have been reported to exhibit time-dependent inhibition [40-43], ginsenosides $R b_{1}(\mathbf{1}), \mathrm{Rb}_{2}(\mathbf{2}), \mathrm{Rc}(\mathbf{3}), \mathrm{Rd}(\mathbf{4}), \mathrm{Rg}_{3}(\mathbf{6}), \mathrm{Rg}_{1}$ (31), Re (32), and Ro (51) did not exhibit any significant timedependent inhibition (Table 5). The ginsenosides also inhibited rat Oatp1b2, exhibiting [with the exception of ginsenoside Ro (51)] $I C_{50}$ values (Supplementary Table S3) greater than the respective values for human OATP1B1/1B3.
Given multiple ginsenosides being simultaneously introduced into and co-circulating in the bloodstream by dosing ShenMai, drug interactions related to ShenMai are expected to result from a joint inhibition of the human transporters by these herbal compounds. Combination of ginsenosides $\mathrm{Rb}_{1}(\mathbf{1}), \mathrm{Rb}_{2}(\mathbf{2}), \mathrm{Rc}$ (3), $\operatorname{Rd}(4)$, $\operatorname{Re}(32)$, and $\operatorname{Ro}(51)$ and that of ginsenosides $R b_{1}(\mathbf{1})$, $\mathrm{Rb}_{2}(\mathbf{2}), \mathrm{Rc}(\mathbf{3})$, and $\mathrm{Rd}(\mathbf{4})$ yielded additive inhibition of OATP1B3 and OATP1B1, as indicated by the combination indices shown in Table 6.

\section{DISCUSSION}

In ShenMai, Hongshen ginsenosides are the major saponins present, while Maidong ophiopogonins are minor. Ginsenosides are also present in medicinal herbs of other Panax species, including $P$. notoginseng roots (Sanqi) and $P$. quinquefolius roots (Xiyangshen) $[35,44]$. In China, both Hongshen and Sanqi are used in intravenous herbal injections, e.g., ShenMai, using Hongshen, and XueShuanTong, using Sanqi. Based on our current and ongoing investigations, differences in ginsenoside composition between ShenMai and XueShuanTong are: presence of greater number of major ppd-type ginsenosides but less number of major ppt-type ginsenosides in ShenMai than in XueShuanTong and presence of ginsenosides of other types in ShenMai but negligible in XueShuanTong. Given that some ppd- and ppt-type ginsenosides exhibit significantly different inhibition potencies on OATP1B transporters [14], and that such inhibition remains to be evaluated for many Hongshen-origin ginsenosides, ShenMai-drug interactions warrant a separate investigation.

Here, high-priority ShenMai ginsenosides were identified in rat pharmacokinetic study, prior to in vitro transporter inhibition study. Also, drug interaction-related pharmacokinetics (including plasma $C_{\max }$ and $t_{1 / 2}$ ) were characterized for the identified 
Table 4. Cellular uptake of ShenMai saponins by rat Oatp1b2, human OATP1B1, and human OATP1B3

\begin{tabular}{|c|c|c|c|c|}
\hline \multirow[t]{2}{*}{ Compounds (ID) } & \multirow{2}{*}{$\begin{array}{l}\text { Transport in } \\
\text { mock cell } \\
\text { (pmol/min/ } \\
\text { mg protein) }\end{array}$} & \multicolumn{3}{|c|}{ Net transport ratio } \\
\hline & & Oatp1b2 & OATP1B1 & OATP1B3 \\
\hline \multicolumn{5}{|l|}{ Ppd-type ginsenosides } \\
\hline Ginsenoside $\mathrm{Rb}_{1}(\mathbf{1})$ & $5.4 \pm 1.6$ & $1.2 \pm 0.5$ & $1.3 \pm 0.2$ & $1.3 \pm 0.1$ \\
\hline Ginsenoside $\mathrm{Rb}_{2}$ (2) & $9.4 \pm 6.0$ & $1.5 \pm 0.3$ & $1.8 \pm 0.1$ & $1.8 \pm 0.1$ \\
\hline Ginsenoside Rc (3) & $6.2 \pm 1.3$ & $2.2 \pm 0.5$ & $1.3 \pm 0.1$ & $1.4 \pm 0.2$ \\
\hline Ginsenoside Rd (4) & $9.8 \pm 2.0$ & $1.4 \pm 0.1$ & $1.5 \pm 0.1$ & $1.2 \pm 0.1$ \\
\hline Ginsenoside $\mathrm{Ra}_{1}$ (5) & $4.4 \pm 0.7$ & $1.4 \pm 0.2$ & $1.4 \pm 0.1$ & $1.5 \pm 0.3$ \\
\hline Ginsenoside $\mathrm{Rg}_{3}(\mathbf{6})$ & $111.9 \pm 47.5$ & $1.5 \pm 0.3$ & $1.4 \pm 0.3$ & $1.8 \pm 0.6$ \\
\hline Ginsenoside $\mathrm{Ra}_{2}$ (7) & $4.2 \pm 0.9$ & $2.5 \pm 0.5$ & $1.8 \pm 0.5$ & $2.0 \pm 0.4$ \\
\hline Ginsenoside $\mathrm{Ra}_{3}(\mathbf{8})$ & $5.4 \pm 1.3$ & $2.6 \pm 1.3$ & $1.1 \pm 0.3$ & $1.0 \pm 0.1$ \\
\hline \multicolumn{5}{|l|}{ Ppt-type ginsenosides } \\
\hline Ginsenoside $\mathrm{Rg}_{1}$ (31) & $1.5 \pm 0.3$ & $7.1 \pm 0.5$ & $2.2 \pm 0.8$ & $11.6 \pm 0.8$ \\
\hline Ginsenoside Re (32) & $2.4 \pm 0.8$ & $78.2 \pm 14.1$ & $2.9 \pm 0.0$ & $24.7 \pm 3.0$ \\
\hline Ginsenoside $\mathrm{Rg}_{2}$ (33) & $3.3 \pm 0.8$ & $20.9 \pm 1.1$ & $8.8 \pm 1.0$ & $18.7 \pm 2.3$ \\
\hline Ginsenoside Rf (34) & $2.1 \pm 0.5$ & $4.4 \pm 0.1$ & $2.3 \pm 0.4$ & $11.0 \pm 1.8$ \\
\hline $\begin{array}{l}\text { Notoginsenoside } \\
R_{1} \text { (35) }\end{array}$ & $1.0 \pm 0.2$ & $18.2 \pm 2.8$ & $3.1 \pm 0.4$ & $13.2 \pm 1.0$ \\
\hline $\begin{array}{l}\text { 20-Glucoginsenoside } \\
\text { Rf (36) }\end{array}$ & $2.1 \pm 0.1$ & $9.4 \pm 1.9$ & $0.6 \pm 0.1$ & $10.7 \pm 1.3$ \\
\hline Ginsenoside $\mathrm{Rh}_{1}$ (37) & $6.6 \pm 2.1$ & $4.8 \pm 0.6$ & $2.5 \pm 0.1$ & $2.1 \pm 0.3$ \\
\hline \multicolumn{5}{|c|}{ Ginsenosides of other types } \\
\hline Ginsenoside Ro (51) & $10.0 \pm 4.9$ & $15.3 \pm 1.4$ & $13.4 \pm 2.4$ & $20.2 \pm 6.0$ \\
\hline Ginsenoside $\mathrm{Rg}_{6}$ (52) & $39.7 \pm 13.6$ & $6.6 \pm 0.7$ & $12.9 \pm 2.0$ & $10.8 \pm 0.7$ \\
\hline Ginsenoside $\mathrm{Rk}_{1}$ (53) & $\begin{array}{l}1063.9 \pm \\
117.0\end{array}$ & $1.5 \pm 0.1$ & $1.8 \pm 0.1$ & $1.9 \pm 0.2$ \\
\hline Ginsenoside $\mathrm{Rh}_{4}$ (54) & $439.8 \pm 49.3$ & $1.3 \pm 0.2$ & $1.8 \pm 0.0$ & $1.3 \pm 0.2$ \\
\hline Ginsenoside $\mathrm{Rg}_{5}$ (55) & $\begin{array}{l}2595.9 \pm \\
215.1\end{array}$ & $1.3 \pm 0.1$ & $1.5 \pm 0.1$ & $1.4 \pm 0.1$ \\
\hline \multicolumn{5}{|l|}{ Ophiopogonins } \\
\hline Ophiopogonin D (71) & $96.4 \pm 15.4$ & $2.7 \pm 0.6$ & $4.9 \pm 0.5$ & $4.0 \pm 1.0$ \\
\hline Ophiopogonin $\mathrm{D}^{\prime}$ (72) & $\begin{array}{l}1900.0 \pm \\
292.6\end{array}$ & $1.4 \pm 0.1$ & $2.4 \pm 0.5$ & $2.0 \pm 0.5$ \\
\hline
\end{tabular}

ginsenosides. Interspecies similarities and differences in these inter-compound differences are considered in using these rat pharmacokinetic data for investigating ShenMai-drug interaction potential. Systemic clearance of ginsenosides generally involves two or more of three elimination routes: hepatobiliary excretion, renal excretion, and hepatic metabolism [14, 26, 45]. Hepatobiliary excretion of ginsenosides is governed mainly by action of the hepatic uptake transporter and their membrane permeability. ShenMai ginsenosides significantly differ in their cellular transport by rat Oatp1b2: ppt-type ginsenosides (31-37), ginsenosides Ro (51) and $\mathrm{Rg}_{6}(\mathbf{5 2})$ are substrates, while the other ginsenosides (1-8 and 53-55) are not. This in vitro transport profile correlates well with the compounds' $\mathrm{CL}_{B}$ profile in rats. Such inter-compound differences in vitro also occur for human OATP1B3 and for human OATP1B1. For most of the ginsenosides that exhibit slow rates of membrane permeation, the inter-compound differences in hepatobiliary excretion in rats depend on inter-compound differences in the transporters' actions. For ginsenosides $\mathrm{Rg}_{3}(\mathbf{6})$, $\mathrm{Rk}_{1}(\mathbf{5 3}), \mathrm{Rh}_{4}(\mathbf{5 4})$, and $\mathrm{Rg}_{5}(\mathbf{5 5})$ that are not substrates of the rat and human transporters, their rapid hepatobiliary excretion is due to their good membrane permeability. Accordingly, hepatobiliary excretion of ShenMai ginsenosides in humans is expected to be similar to that in rats. Renal excretion of most ShenMai ginsenosides is mainly based on glomerular filtration, which is governed by plasma protein binding. Interspecies similarity in such binding suggests that inter-ginsenoside differences in renal excretion in rats can largely be extrapolated to humans. Hepatic metabolism of ShenMai ginsenosides in rats, as suggested by in vitro metabolism data, appears to involve: (1) deglycosylation of ginsenosides $R b_{1}$ (1) and $R b_{2}$ (2) to yield ginsenoside $R d(4)$; and (2) mono-oxidation of ginsenosides $\mathrm{Rg}_{3}(\mathbf{6}), \mathrm{Rg}_{2}(\mathbf{3 3}), \mathrm{Rf}(\mathbf{3 4})$, and $\mathrm{Rh}_{1}$ (37). The in vitro data also suggest that these metabolic reactions could similarly occur in humans. The $V_{S S}$ of ShenMai ginsenosides in rats are $0.2-1.0$ times as much as rat volume of total body water. Because the inter-compound differences in $V_{S S}$ are mainly due to the compounds' differences in plasma protein binding, membrane permeability, and hepatic transportermediated transport, such differences in humans are expected to be similar to those in rats. Our ongoing investigation of XueShuanTong indicates that differences in plasma $C_{\max }$ and $t_{1 / 2}$ among the ginsenosides in rats correlate well with the respective differences in human subjects (Supplementary Fig. S3). Collectively, rat plasma $C_{\max }$ and $t_{1 / 2}$ could be used to predict the contributions of ginsenosides to OATP1B-mediated ShenMaidrug interaction potential in humans. Meanwhile, despite the preceding interspecies similarities, measurement of unbound plasma $C_{\max }$ and $t_{1 / 2}$ of ShenMai ginsenosides in humans is required for estimating ShenMai-drug interaction indices. Interspecies similarities and differences in ShenMai ginsenosides' parameters related to drug interaction are summarized in Supplementary Table S4.

All the circulating ShenMai ginsenosides inhibit human OATP1B3 in vitro, but to varying extents. Although these compounds inhibit OATP1B1 less potently than OATP1B3, intercompound differences in inhibition potency are similar on both transporters (Table 5). Two structural features of ginsenosides appear to favor potent inhibition of the OATP1B transporters, i.e., (1) presence of 20(S)-protopanaxadiol as aglycone and/or (2) absence of sugar attachment at C-20. These two features also seem to enhance the compound's binding in human plasma. The ppd-type ginsenosides (1-8) exhibit potent OATP1B3 inhibition in vitro, with ginsenoside $\mathrm{Rg}_{3}$ (6; the only circulating ppd-type ginsenoside without any sugar attachment at C-20) being the most potent inhibitor. Although such inhibition by ppttype ginsenosides (31-37) is generally weak, the $I C_{50}$ values of ppt-type ginsenosides without any sugar attachment at C-20 (33, 34, and 37) are smaller than those of the other ppt-type ginsenosides (31, 32, 35, and 36). Ginsenosides of other types (52-55), without any sugar attachment at C-20, also exhibit potent OATP1B3 inhibition. Ginsenoside Ro $(\mathbf{5 1})$ has structural features different from the other ShenMai ginsenosides'; it is also a potent OATP1B3 inhibitor. Regarding $\mathrm{IC}_{50}$, inhibition of OATP1B1 was reported to be substrate-dependent for several inhibitors of the transporter; using a sensitive probe substrate can avoid false-negative prediction of OATP1B1-mediated drug interactions $[46,47]$. Little is known about such substrate-dependent inhibition of OATP1B3. In this investigation, $E_{2} 17 \beta G$ was used as substrate, which appears to be a sensitive substrate for many OATP1B inhibitors.

Pharmacokinetic characteristics also influence the ginsenosides' contributions to ShenMai-drug interaction potential. Given that inter-compound differences in these pharmacokinetic characteristics in rats are most likely similar to the respective differences in humans, contributions of individual ginsenosides to ShenMaidrug interaction potential in humans can be predicted based on their $\left(C \times f_{\text {u-plasma }}\right) / I C_{50}$ ratios, for a single dose of ShenMai, and their $\left(C \times R \times f_{\mathrm{u} \text {-plasma }}\right) / / C_{50}$ ratios, for multiple doses of ShenMai. Here, $C$ and $R$ are rat data, while $f_{\text {u-plasma }}$ and $I C_{50}$ are human data (these terms are defined in Data processing subsection). Contributions of the ppd-type ginsenosides (1-8) 
Table 5. In vitro inhibitory activities of ShenMai saponins towards human OATP1B1 and OATP1B3

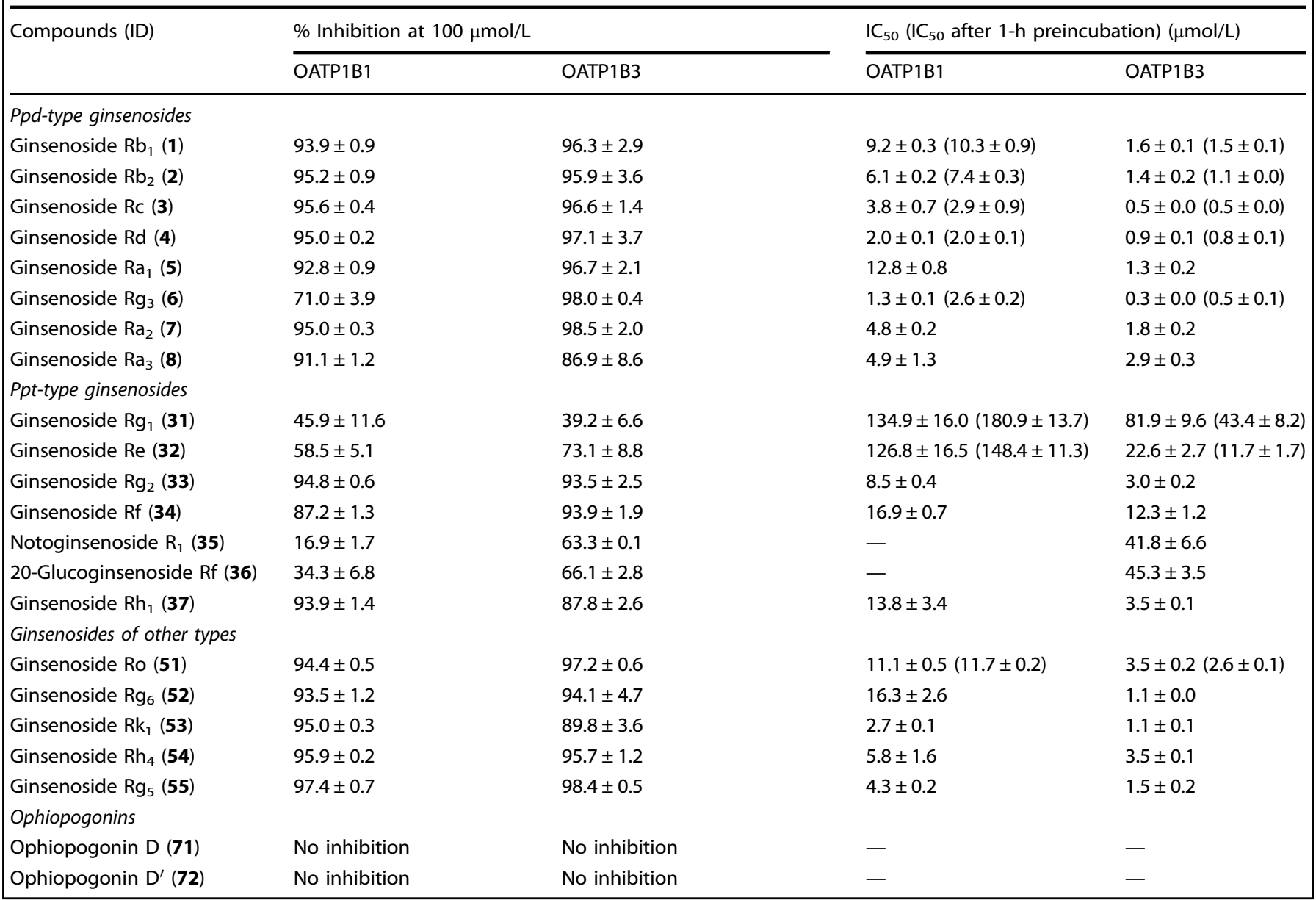

Table 6. Computer-simulated combination indices for ginsenosides $R b_{1}(\mathbf{1}), R b_{2}(\mathbf{2}), R c(\mathbf{3}), R d(\mathbf{4}), \operatorname{Re}(\mathbf{3 1})$, and Ro (51) at different levels of inhibition of human OATP1B1 and OATP1B3

\begin{tabular}{|c|c|c|c|c|c|c|c|c|c|c|}
\hline \multirow[t]{2}{*}{ Percentage inhibition } & \multicolumn{6}{|c|}{$\begin{array}{l}\text { Individual ginsenoside } \\
\text { Compound concentration ( } \mu \mathrm{mol} / \mathrm{L})\end{array}$} & \multicolumn{2}{|c|}{$\begin{array}{l}\text { Ginsenoside combination (compound ratio) } \\
\text { Total concentration ( } \mu \mathrm{mol} / \mathrm{L})\end{array}$} & \multicolumn{2}{|c|}{ Combination index } \\
\hline & 1 & 2 & 3 & 4 & 31 & 51 & $\begin{array}{l}1+2+3+4+31+51 \\
(3: 3: 1: 1: 10: 4)\end{array}$ & $\begin{array}{l}1+2+3+4 \\
(3: 3: 1: 1)\end{array}$ & $\begin{array}{l}1+2+3+ \\
4+31+51\end{array}$ & $1+2+3+4$ \\
\hline \multicolumn{11}{|l|}{ Human OATP1B1 } \\
\hline $50 \%$ & 16 & 20 & 8 & 6 & 392 & 34 & 28 & 14 & 1.0 & 1.1 \\
\hline $75 \%$ & 68 & 41 & 27 & 14 & 1597 & 66 & 65 & 34 & 0.9 & 1.0 \\
\hline $95 \%$ & 763 & 133 & 212 & 52 & 16,893 & 194 & 270 & 158 & 0.9 & 1.0 \\
\hline \multicolumn{11}{|l|}{ Human OATP1B3 } \\
\hline $50 \%$ & 2 & 1 & 0.7 & 0.8 & 27 & 4 & 3 & 1 & 1.2 & 1.0 \\
\hline $75 \%$ & 4 & 4 & 2 & 2 & 85 & 9 & 8 & 3 & 1.1 & 1.0 \\
\hline $90 \%$ & 10 & 10 & 5 & 6 & 269 & 21 & 19 & 9 & 1.0 & 1.0 \\
\hline
\end{tabular}


a (A single dose; OATP1B1)

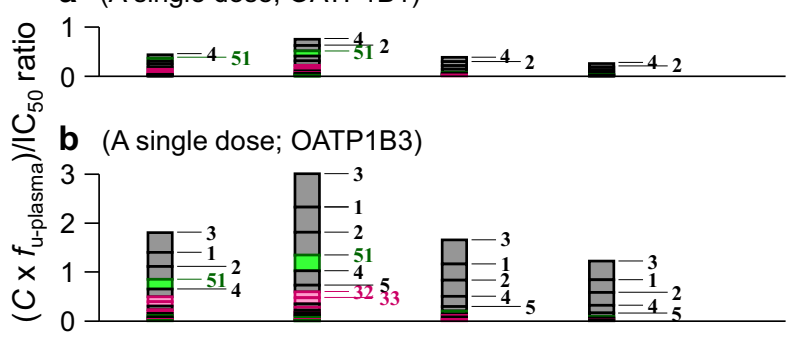

C (Multiple doses at steady state; OATP1B1)

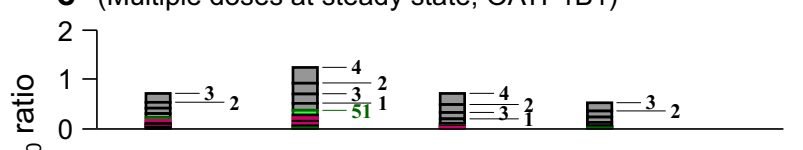

㣢

d (Multiple doses at steady state; OATP1B3)

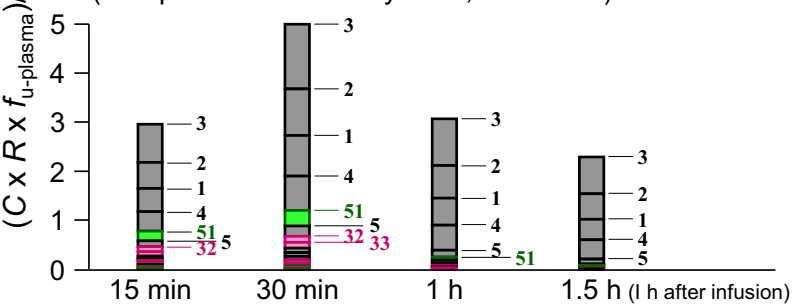

Time after starting a 30-min infusion of ShenMai

Fig. 5 Time-related total $\left(C \times f_{\text {u-plasma }}\right) / I_{50}$ ratios of circulating ginsenosides, for a single dose of ShenMai (a, b), and their timerelated total $\left(C \times R \times f_{\text {u-plasma }}\right) / I_{50}$ ratios, for multiple doses of ShenMai at steady state (c, d). The term $C$ is the plasma concentration of a circulating ginsenoside at 15 and $30 \mathrm{~min}$ and 1 and $1.5 \mathrm{~h}$ after starting a 30-min intravenous infusion of ShenMai in rats or after starting such infusion on the first day of multiple dosing. The terms $R$ (in rats), $f_{\text {u-plasma }}$ (in human plasma), and IC $C_{50}$ (for human OATP1B1 or OATP1B3) are defined in "Data processing" subsection. Data in Table 6 suggested that the ginsenosides jointly inhibited OATP1B3 and OATP1B1 in an additive manner. The numbers in bold to the right of the bars are compound IDs; the associated compound names are shown in Supplementary Tables $\mathrm{S} 5$ and $S 6$, which summarize the $\left(C \times f_{\mathrm{u} \text {-plasma }}\right) / I C_{50}$ ratios of individual circulating ginsenosides, for a single dose of ShenMai, and their time-related total $\left(C \times R \times f_{\mathrm{u} \text {-plasma }}\right) / / C_{50}$ ratios, for multiple doses of ShenMai at steady state. These ratios were estimated to identify ShenMai saponins that were likely to contribute the major part of ShenMai-drug interactions. It is worth mentioning that, although these ratios are not the conventional DDI indices, their estimation was based on the principle for estimating human DDI index and on understanding of similarities and differences, in inter-compound variation, between rats and humans (Tables 3 and 5, Supplementary Tables S3 and S4, and Supplementary Fig. S3). The predicted human DDI indices are shown in Supplementary Table S7; also see Supplementary Method

are limited by their extensive binding in human plasma, despite their potent OATP1B3 inhibition; however, such contributions are enhanced upon repeated doses of ShenMai due to accumulation of these long-circulating compounds. In contrast, contributions of ppt-type ginsenosides (31-37) are limited by their low inhibition potencies, despite their low binding in plasma. Ginsenoside Ro (51) exhibits medium-potent OATP1B3 inhibition and moderate binding in plasma. Contributions of ginsenosides (52-55) are expected to be limited due to their low systemic exposure, despite their potent OATP1B3 inhibition. Due to the inter-compound differences in $t_{1 / 2}$, the contributions of ginsenosides to OATP1B3-mediated ShenMai-drug interaction potential change in a time-related manner: (1) the profile during infusion different from that after terminating the infusion and (2) the profile upon repeated doses different from that after single dose. Figure 5 depicts such time-related profiles for ShenMai (associated data shown in Supplementary Tables S5 and S6), with the individual ginsenosides jointly inhibiting the transporters in an additive manner. Before terminating ShenMai infusion, three types of ginsenosides, i.e, ginsenosides $R c(3), R b_{1}(\mathbf{1}), R b_{2}$ (2), Ro (51), $\operatorname{Rd}(4), \operatorname{Ra}_{1}(5), \operatorname{Re}(\mathbf{3 2})$, and $\operatorname{Rg}_{2}(33)$, contribute $90 \%$ of the OATP1B3-mediated interaction potential. After infusion (one hour after), the ppd-type ginsenosides $(\mathbf{3}, \mathbf{1}, \mathbf{2}, \mathbf{4}$, and 5) alone contribute $93 \%$ of such interaction potential. Similar scenarios took place for OATP1B1, but with lower total inhibition potency. At steady state upon repeated doses of ShenMai, the total ratios of ginsenosides increased significantly due to increased contribution of long-circulating ppd-type ginsenosides, relative to the scenario after a single dose (Fig. 5). It is worth mentioning that intravenous ShenMai is likely to precipitate clinical PK interaction with an OATP1B3 substrate via inhibition of the transporter by multiple ginsenosides. This prediction is based on estimated total DDI indices of ginsenosides in humans after dosing ShenMai using rat-human correlations in $C_{\max }$ and $t_{1 / 2}$ of XueShuanTong ginsenosides (Supplementary Fig. S3); for the details, see Supplementary Method and Table S7.

In summary, an approach for investigating herbal medicinedrug interaction is proposed and illustrated: characterize the medicine's chemical composition, identify multiple herbal compounds that are bioavailable and bioactive for such interaction, and characterize their individual contributions to the interaction and their joint action mode. Twelve potent OATP1B3 inhibitors from circulating ShenMai saponins are reported here for the first time. The inhibitor ginsenosides, except ginsenoside Ro (51), have 20(S)-protopanaxadiol as aglycone and/or no sugar attachment at C-20; they also inhibit OATP1B1 but less potently. OATP1Bmediated ShenMai-drug interactions could result from joint inhibition of the transporters by multiple ginsenosides, among which ginsenosides $R b_{1}(\mathbf{1}), \mathrm{Rb}_{2}(\mathbf{2}), \mathrm{Rc}(\mathbf{3}), \mathrm{Rd}(\mathbf{4}), \mathrm{Ro}(\mathbf{5 1}), \mathrm{Ra}_{1}(\mathbf{5})$, $\operatorname{Re}(32)$, and $\mathrm{Rg}_{2}(\mathbf{3 3})$ are predicted to contribute the major part of such interactions, in an additive and time-related manner. Due to the long-circulating ppd-type ginsenosides, the interaction risk should be monitored even after terminating infusion of ShenMai; such risk can be increased upon repeated doses of the herbal injection. This investigation, as well as a planned human pharmacokinetic study of ShenMai, will inform physiologically based pharmacokinetic model-based prediction and clinical studies of drug interaction with this herbal injection; it will also inform investigation of intra-ShenMai interactions between the OATP1B3-inhibiting ginsenosides and the OATP1B3 substrate ginsenosides.

\section{ACKNOWLEDGEMENTS}

This work was supported in part by the National Natural Science Foundation of China (Grants 81403176 and 81673582 ); by the National Science and Technology Major Project of China "Key New Drug Creation and Manufacturing Program" (Grants 2017ZX09301012-006 and 2009ZX09304-002); and by the Strategic Priority Research Program of the Chinese Academy of Sciences (Grant XDA12050306). Olajide E. Olaleye is a recipient of CAS-TWAS President's PhD Fellowship.

\section{AUTHOR CONTRIBUTIONS}

Participated in research design: $\mathrm{CL}$, J-IY, and OEO. Conducted experiments: OEO, J-IY, WN, F-fD, F-qW, FX, SP, and J-IL. Performed data analysis: CL, J-IY, and OEO. Wrote or contributed to the writing of the manuscript: $\mathrm{CL}, \mathrm{OEO}$, and J-IY.

\section{ADDITIONAL INFORMATION}

The online version of this article (https://doi.org/10.1038/s41401-018-0173-9) contains supplementary material, which is available to authorized users. 


\section{REFERENCES}

1. Zhang M-M, Liu X-M, Li J, He L, Tripathy D. Chinese medicinal herbs to treat the side-effects of chemotherapy in breast cancer patients. Cochrane Database Syst Rev. 2007;18:CD004921.

2. Lam W, Bussom S, Guan F-L, Jiang Z-L, Zhang W, Gullen EA, Liu SH, Cheng YC. The four-herb Chinese medicine PHY906 reduces chemotherapy-induced gastrointestinal toxicity. Sci Transl Med. 2010;2:45ra59.

3. Li X-L, Zhang J, Huang J, Ma A-Q, Yang J-F, Li W-M, et al. A multicenter, randomized, double-blind, parallel-group, placebo-controlled study of the effects of Qili Qiangxin capsules in patients with chronic heart failure. J Am Coll Cardiol. 2013;62:1065-72

4. Hao P-P, Jiang F, Chen Y-G, Yang J-M, Zhang K, Zhang M-X, et al. Traditional Chinese medication for cardiovascular disease. Nat Rev Cardiol. 2015;12:115-22.

5. Chinese Society of Critical Care Medicine. Chinese guidelines for management of severe sepsis and septic shock 2014. Chin Crit Care Med. 2015;27:401-26.

6. Expert Committee on Clinical Application of Glycyrrhizin Preparation in the Treatment of Liver Diseases. Expert consensus on clinical application of glycyrrhizin preparation in the treatment of liver diseases. J Clin Hepatol. 2016;32: 844-52.

7. Song Y-L, Yao C, Shang H-C, Yao X-Q, Bai C-X. Late-breaking abstract: intravenous infusion of Chinese medicine XueBiJing significantly improved clinical outcome in severe pneumonia patients in a multiple center randomized controlled clinical trials. Eur Respir J. 2016:48:OA3323.

8. Fugh-Berman A. Herb-drug interactions. Lancet. 2000;355:134-8.

9. Hermann R, Richter O. Clinical evidence of herbal drugs as perpetrators of pharmacokinetic drug interactions. Planta Med. 2012;78:1458-77.

10. Stieger B, Mahdi ZM, Jager W. Intestinal and hepatocellular transporters: therapeutic effects and drug interactions of herbal supplements. Annu Rev Pharmacol Toxicol. 2017;57:399-416.

11. Grimstein M, Huang S-M. A regulatory science viewpoint on botanical-drug interactions. J Food Drug Anal. 2018. 10.016/j.jfda.2018.01.013.

12. Brantley SJ, Argikar AA, Lin YS, Nagar S, Paine MF. Herb-drug interactions: challenges and opportunities for improved predictions. Drug Metab Dispos. 2014;42: 301-17.

13. Gufford BT, Chen G, Vergara AG, Lazarus P, Oberlies NH, Paine MF. Milk Thistle constituents inhibit raloxifene intestinal glucuronidation: a potential clinically relevant natural product-drug interaction. Drug Metab Dispos. 2015;43:353-9.

14. Jiang R-R, Dong J-J, Li X-X, Du F-F, Jia W-W, Xu F, et al. Molecular mechanisms governing different pharmacokinetics of ginsenosides and potential for ginsenoside-perpetrated herb-drug interactions on OATP1B3. $\mathrm{Br} J$ Pharmacol. 2015;172:1059-73.

15. Wu X, Ma J, Ye Y, Lin G. Transporter modulation by Chinese herbal medicines and its mediated pharmacokinetic herb-drug interactions. J Chromatogr B. 2016;1026: 236-53.

16. Li Y, Revalde J, Paxton JW. The effects of dietary and herbal phytochemicals on drug transporters. Adv Drug Deliv Rev. 2017;116:45-62.

17. Dong J-J, Olaleye $\mathrm{OE}$, Jiang R-R, Li J, Lu C, Du F-F, et al. Glycyrrhizin has a high propensity to be a victim of herb-drug interactions on hepatic OATP1B1/1B3. Br J Pharmacol. 2018;175:3486-503.

18. Xian S-X, Yang Z-Q, Lee J, Jiang Z-P, Ye X-H, Luo L-Y, et al. A randomized, doubleblind, multicenter, placebo-controlled clinical study on the efficacy and safety of Shenmai injection in patients with chronic heart failure. J Ethnopharmacol. 2016;186:136-42

19. Zhang W-L, Yan T-H, Liu B, Wang J, Zhao N-P, Shu L-X, et al. Systematic reviews and meta-analysis of ShenMai injection with chemotherapy on treatment of patients with non-small cell lung cancer. Pract Pharm Clin Remed. 2011;14: 95-101.

20. Fan $\mathrm{X}-\mathrm{H}$, Wang $\mathrm{Y}$, Cheng $\mathrm{Y}-\mathrm{Y}$. LC/MS fingerprinting of Shenmai injection: a novel approach to quality control of herbal medicines. J Pharm Biomed Anal. 2006;40: 591-7.

21. Karmazyn M, Moey M, Gan XT. Therapeutic potential of ginseng in the management of cardiovascular disorders. Drugs. 2011;71:1989-2008.

22. Moey M, Gan XT, Huang CX, Rajapurohitam V, Martínez-Abundis E, Lui EMK et al. Ginseng reverse established cardiomyocytes hypertrophy and postmyocardial infarction-induced hypertrophy and heart failure. Circ Heart Fail. 2012;5:504-14

23. Chen $M-H$, Chen X-J, Wang $M$, Lin L-G, Wang Y-T. Ophiopogon japonicus-a phytochemical, ethnomedicinal and pharmacological review. J Ethnopharmacol. 2016;181:193-213.

24. Zhang A-J, Wang C-Y, Liu Q, Meng Q, Peng J-Y, Sun H-J, et al. Involvement of organic anion-transporting polypeptides in the hepatic uptake of dioscin in rats and humans. Drug Metab Dispos. 2013;41:994-1003.

25. Sheng J-J, Tian X-T, Xu G-L, Wu Z-T, Chen C, Wang L, et al. The hepatobiliary disposition of timosaponin b2 is highly dependent on influx/efflux transporters but not metabolism. Drug Metab Dispos. 2015;43:63-72.
26. Hu Z-Y, Yang J-L, Cheng C, Huang Y-H, Du F-F, Wang F-Q, et al. Combinatorial metabolism notably affects human systemic exposure to ginsenosides from orally administered extract of Panax notoginseng roots (Sanqi). Drug Metab Dispos. 2013:41:1457-69.

27. McGrath J, Drummond G, McLachlan E, Kilkenny C, Wainwright C. Guidelines for reporting experiments involving animals: the ARRIVE guidelines. $\mathrm{Br} J$ Pharmacol. 2010;160:1573-6.

28. Kilkenny C, Browne W, Cuthill IC, Emerson M, Altman DG. Animal research: reporting in vivo experiments: the ARRIVE guidelines. Br J Pharmacol. 2010;160: 1577-9.

29. Reagan-Shaw $S$, Nihal $M$, Ahmad N. Dose translation from animal to human studies revisited. FASEB J. 2008;22:659-61.

30. Guo B, Li C, Wang G-J, Chen L-S. Rapid and direct measurement of free concentrations of highly protein-bound fluoxetine and its metabolite norfluoxetine in plasma. Rapid Commun Mass Spectrom. 2006;20:39-47.

31. Posada MM, Bacon JA, Schneck KB, Tirona RG, Kim RB, Higgins JW, et al. Prediction of renal transporter mediated drug-drug interactions for pemetrexed using physiologically based pharmacokinetic modeling. Drug Metab Dispos. 2015;43:325-34.

32. Chou TC. Theoretical basis, experimental design, and computerized simulation of synergism and antagonism in drug combination studies. Pharmacol Rev. 2006;58:621-81.

33. Chou TC. Drug combination studies and their synergy quantification using the Chou-Talalay method. Cancer Res. 2010;70:440-6.

34. Xie T, Liang Y, Hao H-P, Xie JAL, Gong P, Dai C, et al. Rapid identification of ophiopogonins and ophiopogonones in Ophiopogon japonicus extract with a practical technique of mass defect filtering based on high resolution mass spectrometry. J Chromatogr A. 2012;1227:234-44.

35. Yang W-Z, Ye M, Qiao X, Liu C-F, Miao W-J, Bo T, et al. A strategy for efficient discovery of new natural compounds by integrating orthogonal column chromatography and liquid chromatography/mass spectrometry analysis: Its application in Panax ginseng, Panax quinquefolium and Panax notoginseng to characterize 437 potential new ginsenosides. Anal Chim Acta. 2012;739:56-66.

36. Shin BK, Kwon SW, Park JH. Chemical diversity of ginseng saponins from Panax ginseng. J Ginseng Res. 2015;39:287-98.

37. Li F, Cheng T-F, Dong X, Li P, Yang H. Global analysis of chemical constituents in Shengmai injection using high performance liquid chromatography coupled with tandem mass spectrometry. J Pharm Biomed Anal. 2016;117:61-72.

38. European Medicines Agency. Guideline on bioanalytical method validation London, UK: European Medicines Agency. www.ema.europa.eu/docs/en_GB/ document_library/Scientific_guideline/2011/08/ WC500109686.pdf; 2012.

39. Food and Drug Administration Center for Drug Evaluation and Research In vitro metabolism- and transporter-mediated drug-drug interaction studies guidance for industry (Draft Guidance). Silver Spring, MD: U.S. Food and Drug Administration. www.fda.gov/downloads/Drugs/GuidanceComplianceRegulatorylnformation/Guidances/UCM581965.pdf; 2017.

40. Amundsen R, Christensen H, Zabihyan B, Asberg A. Cyclosporine A, but not tacrolimus, shows relevant inhibition of organic anion-transporting protein 1B1-mediated transport of atorvastatin. Drug Metab Dispos. 2010;38:1499-504.

41. Gertz M, Cartwright CM, Hobbs MJ, Kenworthy KE, Rowland M, Houston JB, et al. Cyclosporine inhibition of hepatic and intestinal CYP3A4, uptake and efflux transporters: application of PBPK modeling in the assessment of drug-drug interaction potential. Pharm Res. 2013;30:761-80.

42. Izumi S, Nozaki Y, Maeda K, Komori T, Takenaka O, Kusuhara H, et al. Investigation of the impact of substrate selection on in vitro organic anion transporting polypeptide 1B1 inhibition profiles for the prediction of drug-drug interactions. Drug Metab Dispos. 2015;43:235-47.

43. Shitara Y, Sugiyama Y. Preincubation-dependent and long-lasting inhibition of organic anion transporting polypeptide (OATP) and its impact on drug-drug interactions. Pharmacol Ther. 2017;177:67-80.

44. Yang W-Z, Qiao X, Li K, Fan J-R, Bo T, Guo D-A, et al. Identification and differentiation of Panax ginseng, Panax quinquefolium and Panax notoginseng by monitoring multiple diagnostic chemical markers. Acta Pharm Sin B. 2016;6: 568-75.

45. Liu H-F, Yang J-L, Du F-F, Gao X-M, Ma X-T, Huang Y-H, et al. Absorption and disposition of ginsenosides after oral administration of Panax notoginseng extract to rats. Drug Metab Dispos. 2009;37:2290-8.

46. Noé J, Portmann R, Brun ME, Funk C. Substrate-dependent drug-drug interactions between gemfibrozil, fluvastatin and other organic anion-transporting peptide (OATP) substrates on OATP1B1, OATP2B1, and OATP1B3. Drug Metab Dispos. 2007:35:1308-14.

47. Izumi S, Nozaki Y, Komori T, Maeda K, Takenaka O, Kusano K, et al. Substratedependent inhibition of organic anion transporting polypeptide 1B1: comparative analysis with prototypical probe substrates estradiol-17 $\beta$-glucuronide, estrone-3sulfate, and sulfobromophthalein. Drug Metab Dispos. 2013;41:1859-66. 